\title{
The oxygen abundance gradients in the gas discs of galaxies in the EAGLE simulation
}

\author{
Patricia B. Tissera ${ }^{1 \star}$, Yetli Rosas-Guevara ${ }^{1,2}$, Richard G. Bower ${ }^{3}$, Robert A. Crain ${ }^{4}$, \\ Claudia del P. Lagos ${ }^{5,6}$, Matthieu Schaller ${ }^{7}$, Joop Schaye ${ }^{7}$, Tom Theuns ${ }^{3}$. \\ ${ }^{1}$ Departamento de Ciencias Fisicas, Universidad Andres Bello, 700 Fernandez Concha, Las Condes, Santiago, Chile. \\ 2 Centro de Estudios de Física del Cosmo de Aragón, Plaza San Juan 1, Planta 2, E-44001 Teruel, Spain. \\ ${ }^{3}$ Insitute of Computational Cosmology, Physics Department, University of Durham, South Road, Durham DH1 3LE, UK. \\ ${ }^{4}$ Astrophysics Research Institute, Liverpool John Moores University, 146 Brownlow Hill, Liverpool L3 5RF, UK . \\ ${ }^{5}$ International Centre for Radio Astronomy Research, University of Western Australia, 35 Stirling Highway, Crawley, WA 6009, Australia. \\ ${ }^{6}$ ARC Centre of Excellence for All Sky Astrophysics in 3 Dimensions (ASTRO 3D). \\ ${ }^{7}$ Leiden Observatory, Leiden University, PO Box 9513, 2300 RA, Leiden, the Netherlands.
}

Accepted XXX. Received YYY; in original form ZZZ

\begin{abstract}
We use the EAGLE simulations to study the oxygen abundance gradients of gas discs in galaxies within the stellar mass range $\left[10^{9.5}, 10^{10.8}\right] \mathrm{M}_{\odot}$ at $z=0$. The estimated median oxygen gradient is $-0.011 \pm 0.002 \mathrm{dex} \mathrm{kpc}^{-1}$, which is shallower than observed. No clear trend between simulated disc oxygen gradient and galaxy stellar mass is found when all galaxies are considered. However, the oxygen gradient shows a clear correlation with gas disc size so that shallower abundance slopes are found for increasing gas disc sizes. Positive oxygen gradients are detected for $\approx 40$ per cent of the analysed gas discs, with a slight higher frequency in low mass galaxies. Galaxies that have quiet merger histories show a positive correlation between oxygen gradient and stellar mass, so that more massive galaxies tend to have shallower metallicity gradients. At high stellar mass, there is a larger fraction of rotational-dominated galaxies in low density regions. At low stellar mass, non-merger galaxies show a large variety of oxygen gradients and morphologies. The normalization of the disc oxygen gradients in non-merger galaxies by the effective radius removes the trend with stellar mass. Conversely, galaxies that experienced mergers show a weak relation between oxygen gradient and stellar mass. Additionally, the analysed EAGLE discs show no clear dependence of the oxygen gradients on local environment, in agreement with current observational findings.
\end{abstract}

Key words: galaxies: abundances, galaxies: evolution, cosmology: dark matter

\section{INTRODUCTION}

In the Local Universe, galaxy discs are known to have oxygen abundance gradients in their interstellar medium (ISM) that are (negative) steeper with decreasing luminosity (e.g. Lequeux et al. 1979; Zaritsky et al. 1994) and stellar mass (e.g. Sánchez et al. 2013, 2014; Ho et al. 2015), i.e. the central abundances are larger than those in the outer regions. However, recent results from the MaNGA survey show a weaker relation between metallicity gradients and stellar mass (Belfiore et al. 2017), with a trend for smaller and higher mass galaxies to have flatter metallicity gradients than reported in previous observations. This apparent tension between results obtained from different surveys and datasets shows that we are still far from know-

^ E-mail: patricia.tissera@unab.cl ing the relation between metallicity gradients and stellar mass. With increasingly available data, it became clear that there is a large variety of metallicity gradients as a function stellar mass. This is also the case at high redshift where galaxies are reported to have flat/positive (e.g. Queyrel et al. 2012; Troncoso et al. 2014) as well as negative (e.g. Swinbank et al. 2012; Jones et al. 2013; Yuan et al. 2011) abundance gradients (Carton et al. 2018). An important issue to consider is that the determination of abundances is very sensitive to the adopted metallicity indicator and the spectral resolution (e.g. Kewley \& Ellison 2008; Marino et al. 2013; Mast et al. 2014), so caution is always advised when comparing results from different surveys.

The formation of galaxies in the current cosmological paradigm is a complex and non-linear process (White \& Rees 1978). As gas cools and collapses within the potential well of galaxies, stars form and evolve, injecting 
energy and/or chemical elements into the ISM at different stages of evolution. The new-born stars lock part of the enriched material into long-lived stars while the rest is mixed into the ISM. Within this context, if galaxy formation proceeds in an inside-out fashion, negative metallicity gradients are naturally generated (e.g. Prantzos \& Boissier 2000). However, other mechanisms can alter or modify the metallicity gradients such as galactic fountains, gas inflows (e.g. Amorín et al. 2012; Mollá et al. 2016), galaxy-galaxy interactions (e.g. Rupke et al. 2010; Sillero et al. 2017), rampressure, tidal stripping, amongst others. Together they imprint chemo-dynamical patterns which can be used to unveil the main processes responsible for their origin and evolution (e.g. Bland-Hawthorn \& Freeman 2003).

Hydrodynamical simulations are a powerful tool to understand how metallicity gradients arise and evolve in disc galaxies, since the chemical enrichment of baryons can be tracked as galaxies are assembled in a cosmological context (e.g. Mosconi et al. 2001; Kawata \& Gibson 2003; Kobayashi et al. 2007; Wiersma et al. 2009b). The implementation of baryonic physics relies on subgrid modeling with free parameters that need to be calibrated. Hence, the confrontation of the simulated chemical abundances to observations not only provides a mean to understand their origin, but also a stringent route to set constraints on the models (e.g. Tissera et al. 2012; Gibson et al. 2013; Aumer et al. 2013). Important results, which are complementary to those of numerical simulations, are provided by analytical chemo-dynamical (e.g. Matteucci \& Greggio 1986; Mollá et al. 1997; Chiappini et al. 2001) and semianalytical models (e.g. Cora 2006; De Lucia et al. 2014). These approaches yield negative metallicity gradients in discs if the gas cools and collapses while conserving the angular momentum (Calura et al. 2012; Mollá et al. 2017).

Previous studies using numerical simulations agree on the fact that an inside-out disc formation scenario leads to negative metallicity gradients (e.g Calura et al. 2012; Gibson et al. 2013; Tissera et al. 2016). Pilkington et al. (2012) find that the radial dependence of the star formation efficiency as a function of time sets the metallicity gradients for a given subgrid physics. Enhanced SN feedback and high gas fraction have been shown to be related to the setting of positive metallicity slopes as gas can be ejected from the inner regions where the star formation activity tends to be stronger (e.g. Gibson et al. 2013; Ma et al. 2017). Tissera et al. (2016) report that their simulated gaseous discs show a correlation between metallicity gradients and stellar masses that agrees with the observational results so that smaller galaxies tend to show a larger variety of metallicity slopes (Ho et al. 2015).

Positive metallicity slopes (i.e. lower central abundances than in the outskirts) in the ISM of discs are generally reported to be asociated to galaxy-galaxy interactions in observations (e.g. Molina et al. 2017) as well as in simulations (e.g. Rupke et al. 2010; Tissera et al. 2016; Ma et al. 2017). Observations have shown that the central metallicities of galaxies in close interactions are lower than those in isolation, at least during certain stages of evolution (Kewley et al. 2006; Ellison et al. 2008; Michel-Dansac et al. 2008; Di Matteo et al. 2009; Kewley et al. 2010). Numerical simulations find that mergers and interactions are efficient mechanisms to trigger in- ward gas inflows (e.g. Barnes \& Hernquist 1996; Tissera 2000; Pedrosa \& Tissera 2015; Lagos et al. 2018). This gas inflows can dilute the central gas metallicities if disc galaxies have initially negative metallicity gradients. In fact, hydrodynamical simulations find a clear trend for galaxies in close pairs to exhibit lower central metallicity compared to galaxies in isolation during the first passage but then, the increase of gas in the central regions triggers new starbursts which, in turn, enrich the ISM and regenerate negative profiles (Perez et al. 2006; Rupke et al. 2010; Perez et al. 2011; Sillero et al. 2017) .

Until recently the simulated samples that have been used to study metallicity gradients have been limited to those of galaxies selected from small cosmological volumes or zoom-in systems. The EAGLE project (Schaye et al. 2015; Crain et al. 2015) provides an opportunity to study a large simulated sample of metallicity gradients in a cosmological context. EAGLE galaxies have been shown to reproduce a variety of observational relations at different scales such as the colour-magnitude relations, the neutral gas content or the clustering properties (e.g. Trayford et al. 2015; Lagos et al. 2015; Artale et al. 2017; Crain et al. 2017). The mass-size relation is reproduced as part of the subgrid calibration of the EAGLE simulations at $z=0$ (Schaye et al. 2015). These simulations are also able to reproduce the observed size evolution (Furlong et al. 2015).

Regarding disc formation, Zavala et al. (2016) show that the stellar disc components in EAGLE preserve their specific angular momentum content as they formed from gas that conserved the specific angular momentum, in global agreement with the standard disc formation model (Fall \& Efstathiou 1980). If bulges and discs are considered together then a net angular momentum loss is expected since stars in the spheroidal components formed from gas that lost specific angular momentum (Obreja et al. 2013; Pedrosa \& Tissera 2015; Stevens et al. 2017; Lagos et al. 2018). Due to the complex assembly history of the structure in a hierarchical scenario, galaxies are subject to different processes that can redistribute the angular momentum content of baryons such as mergers, tidal stripping, bar formation, outflows and stellar migration. Therefore, it is the net balance between losses and gains by the different galaxy components that finally matters (e.g. Sales et al. 2012; Pedrosa \& Tissera 2015; Genel et al. 2018).

The relation between stellar mass, metallicity and star formation in EAGLE simulations has been analysed by De Rossi et al. (2017). These authors find results in global agreement with observations, although a weaker redshift evolution of the mean abundances as a function of stellar mass is reported. The $\alpha$-abundances of massive galaxies are analysed by Segers et al. (2016), showing that observed trends are globally reproduced. Mackereth et al. (2018) examine the elemental abundances of the disc stars of EAGLE's $L^{\star}$ galaxies, finding that abundance patterns similar to those revealed by the SDSS-III/APOGEE survey (see e.g. Hayden et al. 2015) are uncommon. They conclude that the Milky Way's abundances and formation history are unlikely to be representative of the broader population of similarly massive, disc-dominated galaxies.

In this paper, we focus on the analysis of the oxygen abundance gradients of the star-forming gas in the discs of central galaxies identified in the $100 \mathrm{Mpc}$ volume EA- 
GLE simulation (Ref-L100N1504) at $z=0$. This simulation includes the effects of stellar and Active Galactic Nucleus (AGN) feedback. Our EAGLE galaxy sample comprises 592 galaxies with discs that are defined by at least 1000 baryonic particles. The analysed galaxies covered a wide range of morphologies and are located in different local environments.

This paper is organised as follows. In Section 2 we describe the main characteristics of the simulations and the galaxy sample. In Section 3 we discuss the metallicity gradients and SFR of the simulated disc galaxies and confront them with observations. In Section 4 we explore the physical mechanisms determining the metallicity gradients. Our main findings are summarised in Section 5. Appendix A.1. and A.2 discuss the impact of numerical resolution and the impact of varying the SN feedback.

\section{THE EAGLE SIMULATIONS}

We use cosmological simulations that are part of the EAGLE project ${ }^{1}$, which comprises a set of cosmological hydrodynamical simulations with variations in volume, numerical resolution and galaxy formation subgrid models. Here, we only give a brief overview of the simulations and subgrid physics modelling. Extensive descriptions can found in Schaye et al. (2015) and Crain et al. (2015). The EAGLE simulations were carried out with a version of the parallel hydrodynamic code GADGET-3 (Springel 2005). The modifications to the hydrodynamic solver and time stepping are referred to as ANARCHY (Schaller et al. 2015).

The initial conditions are consistent with the Planck Cosmology parameters (Planck Collaboration et al. 2014): $\Omega_{\Lambda}=0.693, \Omega_{\mathrm{m}}=0.307, \Omega_{\mathrm{b}}=0.04825, \sigma_{8}=0.8288$, $h=0.6777, \mathrm{n}_{s}=0.9611$ and $Y=0.248$ where $\Omega_{\Lambda}, \Omega_{\mathrm{m}}$ and $\Omega_{\mathrm{b}}$ are the average densities of matter, dark energy and baryonic matter in units of the critical density $z=0, \sigma_{8}$ is the square root of the linear variance, $h$ is the Hubble parameter $\left(H_{O} \equiv h 100 \mathrm{kms}^{-1}\right), n_{s}$ is the scalar power-law index of the power spectrum of primordial perturbations, and $Y$ is the primordial mass fraction of helium. The subgrid physics parameters was calibrated to reproduce the galaxy mass function at $z=0.1$ and the observed sizes of the galaxies today in the so-called reference model (Schaye et al. 2015). In addition, other variations in the subgrid physics have been explored as presented in Crain et al. (2015).

The largest EAGLE simulation (Ref-L100N1504) has a comoving cubic volume of $100 \mathrm{Mpc}$ in linear extent. The setup of the initial conditions provides a mass resolution of $9.7 \times 10^{6} \mathrm{M}_{\odot}$ for dark matter and an initial mass of $1.81 \times$ $10^{6} \mathrm{M}_{\odot}$ for baryonic particles. The gravitational calculations between particles are computed with a Plummer equivalent softening length of 2.66 comoving kpc limited to a maximum physical size of $0.70 \mathrm{kpc}$. In Table 1 , we summarise the main parameters of the simulated used in this work.

1 The reader is referred to http://eaglesim.org, http://eagle.strw.leidenuniv.nl for a global description of the project, access to movies and images and to the database of galaxies are described in McAlpine et al. (2016).

\subsection{Subgrid Physics}

In this section, we summarise the main characteristics of the subgrid modeling. Full details and discussions are given by Schaye et al. (2015). The radiative cooling and photoheating implementations are described in Wiersma et al. (2009a). The radiative rates are calculated on an element-by-element basis for an ionised gas in equilibrium in the presence of an ionising UV/X-Ray background (model of Haardt \& Madau 2001) and the Cosmic Microwave Background. Eleven elements $(\mathrm{H}, \mathrm{He}, \mathrm{N}, \mathrm{O}, \mathrm{C}, \mathrm{Ne}, \mathrm{Mg}, \mathrm{Si}, \mathrm{S}, \mathrm{Ca}$, and $\mathrm{Fe})$ are tracked individually, with yields described by Wiersma et al. (2009b). The radiative cooling and heating rates are computed with the software Cloudy (Ferland et al. 2013). Collisional ionisation equilibrium and non-ionising background are assumed prior to reionization.

Star formation is implemented stochastically following the model of Schaye \& Dalla Vecchia (2008), accounting for a metallicity-dependent star-formation density threshold, $n_{\mathrm{z}}^{*}$ (Schaye et al. 2010), of

$n_{\mathrm{z}}^{*}=10^{-1} \mathrm{~cm}^{-3}\left(\frac{Z}{0.002}\right)^{-0.64}$,

to capture the transition from a warm, atomic to a cold, molecular phase (Schaye 2004). The star formation rate reproduces the empirical Schmidt-Kennicutt law which is written in terms of a pressure law. A pressure floor is assumed as a function of density, $P \propto \rho^{\gamma \mathrm{eff}}$, for gas with $\gamma_{\mathrm{eff}}=4 / 3$. With this value of $\gamma_{\mathrm{eff}}$, the Jeans mass, and the ratio of the Jeans length to the SPH kernel length are independent of density, preventing spurious fragmentation due to a lack of resolution. Star-forming gas particles are stochastically selected to become star particles which represent a simple stellar population formed with a Chabrier (2003) Initial Mass Function.

Stellar mass losses and metal enrichment due to stellar evolution are modeled as described in Wiersma et al. (2009b). The model tracks the nucleosynthesis and enrichment of the 11 elements mentioned above by three evolutionary channels: (1) AGB stars, (2) supernova (SNe) type Ia and (3) massive stars and core-collapse SNe. Stellar evolutionary tracks and yields follow Portinari et al. (1998), Marigo (2001) and Thielemann et al. (1993). The abundance evolution is sensitive to the particular choice of yields.

The stellar feedback is treated stochastically, following the thermal injection method described in Dalla Vecchia \& Schaye (2012). The fraction of the available energy from core-collapse $\mathrm{SNe}, f_{\text {th }}$, injected into the ISM depends on the local metallicity and density as

$f_{\mathrm{th}}=f_{\mathrm{th}, \min }+\frac{f_{\mathrm{th}, \text { max }}-f_{\mathrm{th}, \text { min }}}{1+\left(\frac{Z}{0.1 Z_{\odot}}\right)^{n_{Z}}\left(\frac{n_{\mathrm{H}, \mathrm{birh}}}{n_{\mathrm{H}, 0}}\right)^{-n_{\mathrm{n}}}}$,

where $Z$ is the metallicity, $n_{\mathrm{H} \text {,birth }}$ is the density of the star particle inherited from the parent gas particle and $Z_{\odot}=0.0127$ is the solar metallicity. The parameters $n_{\mathrm{H}, 0}$ and $n_{\mathrm{n}}$ are calibrated to reproduce the galaxy mass function at $z=0.1$ and the sizes of galaxies today. The amount of energy available is injected $30 \mathrm{Myr}$ after the birth of the stellar population. Neighbouring gas particles are stochastically selected to be heated by a fixed temperature difference of $\Delta T=10^{7.5} \mathrm{~K}$.

Black holes with mass $m_{\text {seed }}=1.48 \times 10^{5} \mathrm{M}_{\odot}$ are placed in the centres of haloes with mass greater than $1.48 \times 10^{10} \mathrm{M}_{\odot}$, 
using the BH seeding method of Springel (2005). Black holes can grow through merger events and gas accretion. The accretion rates are computed as the modified Bondi-Hoyle accretion rate of Rosas-Guevara et al. (2015) and Schaye et al. (2015). To modulate the Bondi- Hoyle accretion rate in high circulation flows, a viscosity parameter is introduced. The accretion rates are also limited by the Eddington rate. Similar to the feedback from star formation, AGN feedback is treated stochastically and thermally. The AGN feedback parameters have been calibrated to reproduce the stellar mass galaxy function and the scaling relation between stellar mass and the mass of the central black hole for galaxies observed in the Local Universe.

\subsection{The simulated galaxies}

In this work, we analyse the disc components of central galaxies selected from the Ref-L100N1504 simulation by using the SUBFIND algorithm (Springel 2005; Dolag et al. 2009). The simulated galaxies are decomposed dynamically into a bulge and a disc component following the method and criteria described by Tissera et al. (2012). The stellar (gas) discs are defined by star (gas) particles with $\epsilon=$ $J_{\mathrm{Z}} / J_{\mathrm{Z}, \max }(E)>0.5$, where $J_{\mathrm{Z}, \max }(E)$ is the maximum angular momentum along the main axis of rotation, $J_{\mathrm{Z}}$, over all particles at a given binding energy, $E$.

Only those galaxies that have more than 1000 baryonic particles and more than 100 star-forming gas particles in the disc components are considered for the estimations of the metallicity profiles. The final EAGLE sample comprises 592 gas discs.

A set of properties of the selected galaxies are estimated such as the star formation rate (SFR) and specific star formation rate (sSFR), the disc stellar half-mass radius $\left(\mathrm{R}_{\mathrm{eff}}^{\text {stars }}\right)$, the half-mass radius of the gaseous discs $\left(\mathrm{R}_{\mathrm{eff}}^{\mathrm{gas}}\right)$ and the total half-stellar mass radius $\left(\mathrm{R}_{\mathrm{eff}}^{\mathrm{T}}\right)$. These parameters are estimated by considering particles within $1.5 r_{\mathrm{gal}}{ }^{2}$. The stellar mass of the bulge and disc components identified by the dynamical decomposition described above are used to define the stellar disc-to-total mass ratio, D/T. The EAGLE simulation Ref-L100N1504 yields galaxies with large variety of galaxy morphologies. We analysed the metallicity gradients of the discs components regardless of galaxy morphology. When needed, we assume $\mathrm{D} / \mathrm{T}=0.5$ to separate rotationdominated from dispersion-dominated galaxies.

To determine the metallicity profiles of the simulated gas disc components, we use the oxygen abundances of the star-forming gas. The oxygen profiles are weighted by the star formation rate of the contributing regions to improve the realism of the comparison with observations. A linear regression is fitted within the radial range $[0.5,2] \mathrm{R}_{\mathrm{eff}}^{\text {stars }}$ to estimate the slope $\left(\nabla_{(\mathrm{O} / \mathrm{H})}\right)^{3}$. For illustration purposes, we

\footnotetext{
2 The galaxy radius $r_{\text {gal }}$ is defined as the radius that enclosed 83 per cent of the stellar mass of a system identified by the SUBFIND algorithm (Springel 2005). This radius mimics the isophotal 25 assuming a $\mathrm{M} / \mathrm{L}=1$ (e.g. Tissera et al. 2010).

3 Note that the metallicity dispersion at a given radius is quite large $(\sim$ dex $)$, probably due to the stochasticity related with the star formation and feedback modelling and inefficient metalmixing within the simulated interstellar medium.
}

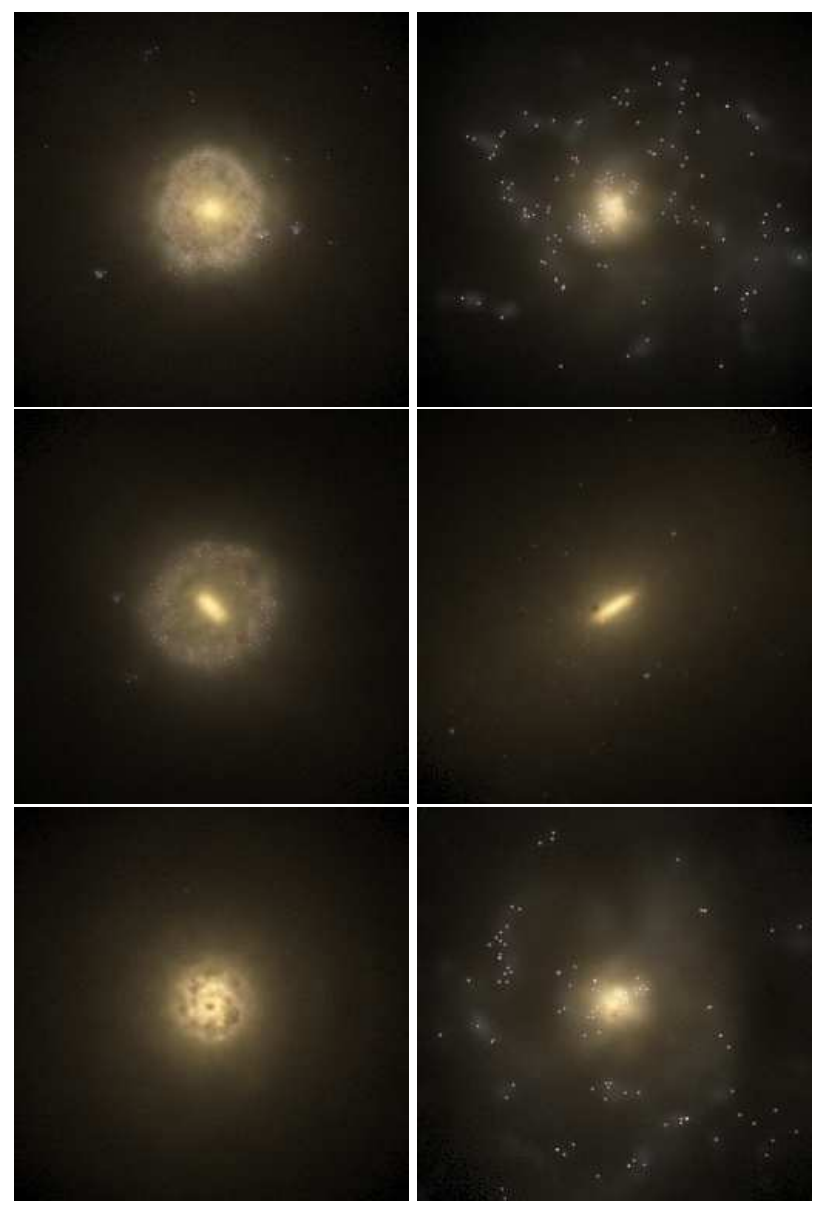

Figure 1. Face-on gri-band mock images of galaxies with negative (left panels) and positive (right panels) gas-phase oxygen gradients of their disc components. Images are taken for the Ref-L100N1504 simulation from the EAGLE database McAlpine et al. (2016).

show in Fig. 1 examples of the gri-band mock images of disc galaxies with positive (right panels) and negative (left panels) metallicity gradients taken from the EAGLE database (McAlpine et al. 2016) and as described in Trayford et al. (2017).

A local galaxy density indicator is calculated to analyse the metallicity gradients as a function of the environment using the count-in-cell (CC) method. The simulated volume is divided into cubic volume cells of $0.5 \mathrm{Mpc}$ on a side. Then, the number of galaxies with stellar mass $\left(\mathrm{M}_{\mathrm{star}}\right)$ larger than $10^{9} \mathrm{M}_{\odot}$ in each cell is counted, defining $N_{\mathrm{CC}}$. This method provides similar results to the fifth neighbour density estimators. We note that similar trends are found when using the dark matter haloes instead. Low-density and high-density environments are defined by adopting $N_{\mathrm{CC}}=2$ as threshold. Most of the analysed disc galaxies are located in lowdensity regions (84 per cent). The rest of these galaxies are distributed in groups with 3 to 23 galaxy members.

As can be seen in the upper panel of Fig. 2, the EAGLE galaxies are able to reproduce the observed morphologydensity dependence with most of the dispersion-dominated galaxies located in groups (Dressler 1980). Recall that we analyse the $\nabla_{(\mathrm{O} / \mathrm{H})}$ of gas discs in central galaxies of different morphological types (i.e. different $\mathrm{D} / \mathrm{T}$ ratios). 
Table 1. Main properties of the analysed EAGLE simulations and the values of the relevant subgrid parameters that differ between them. Columns show: (1) name, (2) box size, (3) initial baryonic particle number (the same number of dark matter particles was used), (4) initial baryonic and (5) dark matter particle mass, (6) comoving and (7) maximum proper gravitational softening, 8) $n_{\mathrm{H}, 0}$ and (9) $n_{n}$ determine the characteristic density and the power law, respectively, of the density dependence of the available SN energy feedback from star formation (see Eq. 2), (10) $f_{\mathrm{th}, \min }$ and (11) $f_{\mathrm{th}, \max }$ are, respectively, the asymptotic minimum and maximum values of the fraction of energy feedback from star formation (see Eq. 2).

\begin{tabular}{llccccccccc}
\hline Name & $\begin{array}{l}\mathrm{L} \\
{[\mathrm{cMpc}]}\end{array}$ & $N$ & $\begin{array}{c}m_{\mathrm{g}} \\
{\left[\mathrm{M}_{\odot}\right]}\end{array}$ & $\begin{array}{c}m_{\mathrm{DM}} \\
{\left[\mathrm{M}_{\odot}\right]}\end{array}$ & $\begin{array}{c}\epsilon_{\mathrm{com}} \\
{[\mathrm{ckpc}]}\end{array}$ & $\begin{array}{c}\epsilon_{\text {prop }} \\
{[\mathrm{pkpc}]}\end{array}$ & $\begin{array}{c}n_{\mathrm{H}, 0} \\
{\left[\mathrm{~cm}^{-3}\right]}\end{array}$ & $n_{n}$ & $f_{\text {th, min }}$ & $f_{\text {th, max }}$ \\
\hline Ref-L100N1504 & 100 & $1504^{3}$ & $1.81 \times 10^{6}$ & $9.70 \times 10^{6}$ & 2.66 & 0.70 & 0.67 & $2 / \ln 10$ & 0.3 & 3.0 \\
Ref-L025N0752 & 25 & $752^{3}$ & $2.26 \times 10^{5}$ & $1.21 \times 10^{6}$ & 1.33 & 0.35 & 0.67 & $2 / \ln 10$ & 0.3 & 3.0 \\
Recal-L025N0752 & 25 & $752^{3}$ & $2.26 \times 10^{5}$ & $1.21 \times 10^{6}$ & 1.33 & 0.35 & 0.25 & $1 / \ln 10$ & 0.3 & 3.0 \\
WeakFB-L025N0376 & 25 & $376^{3}$ & $1.81 \times 10^{6}$ & $9.70 \times 10^{6}$ & 2.66 & 0.70 & 0.67 & $2 / \ln 10$ & 0.15 & 1.5 \\
StrongFB-L025N0376 & 25 & $376^{3}$ & $1.81 \times 10^{6}$ & $9.70 \times 10^{6}$ & 2.66 & 0.70 & 0.67 & $2 / \ln 10$ & 0.6 & 6.0 \\
\hline
\end{tabular}

The middle panel of Fig. 2 displays the distribution of the galaxy stellar masses in the two defined local environments. As expected, there is a trend for higher stellar mass galaxies to be located in the high-density environments while less masive ones tend to be in low-density regions. Unfortunately, there is only a narrow stellar mass range that is sampled simultaneously in both local environments. Hence, our estimations should be taken only as indicative.

In the bottom panel of Fig. 2, the $\nabla_{(\mathrm{O} / \mathrm{H})}$ distributions are shown as a function of local environment. There is no significant statistical difference between them, although a small displacement of the $\nabla_{(\mathrm{O} / \mathrm{H})}$ to more positive values can be seen in low density regions. Gaussian fits to the $\nabla_{(\mathrm{O} / \mathrm{H})}$ distributions yield mean values of $-0.007 \mathrm{dex} \mathrm{kpc} \mathrm{k}^{-1}$ and -0.003 dex kpc $\mathrm{kp}^{-1}$ (widths of $0.022 \mathrm{dex} \mathrm{kpc}^{-1}$ ) for the high-and-lowdensity subsamples, respectively. The bootstrap medians are $-0.009 \pm 0.002 \mathrm{dex} \mathrm{kpc}^{-1}$ and $-0.026 \pm 0.005 \mathrm{dex} \mathrm{kpc}^{-1}$ for galaxies in low-and-high-density regions, respectively. The median $\nabla_{(\mathrm{O} / \mathrm{H})}$ for the whole disc samples is $-0.011 \pm 0.002$ dex $\mathrm{kpc}^{-1}$. The differences between the medians and the Gaussian fit estimations are due to the existence of a tail towards more negative $\nabla_{(\mathrm{O} / \mathrm{H})}$ in high-density regions, which are not well represented by a Gaussian fit.

\section{DISC METALLICITY GRADIENTS}

In Fig. 3, we show $\nabla_{(\mathrm{O} / \mathrm{H})}$ of the gas discs as a function of the stellar mass of the selected EAGLE galaxies. As can be seen, there is no clear trend between the $\nabla_{(\mathrm{O} / \mathrm{H})}$ and $\mathrm{M}_{\text {star }}$. The simulated median $\nabla_{(\mathrm{O} / \mathrm{H})}$ are slightly above the observational values reported for galaxies in the Local Universe (black symbols). In particular, we estimate an offset of $\sim-0.02 \mathrm{dex} \mathrm{kpc}^{-1}$ at $\sim 10^{10.5} \mathrm{M}_{\odot}$ with respect to the MaNGA results reported by Belfiore et al. (2017, see also Appendix A.1 for a discussion on the robustness of this result against numerical resolution). The simulated relation shows more negative metallicity gradients at the low and the higher stellar-mass ends where the scatter increases. From Fig. 3, we can see that there is a significant fraction of oxygen profiles with positive $\nabla_{(\mathrm{O} / \mathrm{H})}$ in the EAGLE discs (i.e. lower central abundances). We find 42 per cent and 30 per cent of positive $\nabla_{(\mathrm{O} / \mathrm{H})}$ in galaxy discs located in low-and-high density regions, respectively. Discs with positive oxygen slopes also contribute to establish a weak mean trend with stellar mass by increasing the scatter of the $\nabla_{(\mathrm{O} / \mathrm{H})}$ at a given stellar mass. This trend seems at odds with some observations (e.g. Zaritsky et al. 1994; Rupke et al. 2010; Ho et al. 2015). However, recent results by Belfiore et al. (2017) from MaNGA survey show a significant contribution of positive abundance slopes. We note that these authors also find an increase of the median $\nabla_{(\mathrm{O} / \mathrm{H})}$ for low and high stellar-mass galaxies that is not detected in the EAGLE discs.

In the left panel of Fig. 4, we show $\nabla_{(\mathrm{O} / \mathrm{H})}$ as a function of galaxy size, considering $\mathrm{R}_{\mathrm{eff}}^{\mathrm{T}}$ (magenta line), $\mathrm{R}_{\mathrm{eff}}^{\text {star }}$ (green line) and $\mathrm{R}_{\text {eff }}^{\text {gas }}$ (cyan line). As can be appreciated from the figure, there is a clear correlation between $\nabla_{(\mathrm{O} / \mathrm{H})}$ and $\mathrm{R}_{\text {eff }}^{\text {gas }}$ so that more negative $\nabla_{(\mathrm{O} / \mathrm{H})}$ are found in galaxies with more compact gas discs. The scatter of the relation increases for decreasing disc size. Conversely, discs with positive $\nabla_{(\mathrm{O} / \mathrm{H})}$ show no trend with $\mathrm{R}_{\text {eff }}^{\text {gas }}$ (black dashed line). Hence, the correlation is set only by discs with negative $\nabla_{(\mathrm{O} / \mathrm{H})}$. This trend can be interpreted as the results of an inside-out disc formation where the systems grow outwards while the star formation moves progressively to the disc outskirts, enriching the ISM and flattening the abundance profiles. The larger the gas discs, the flatter the metallicity gradients (Prantzos \& Boissier 2000), if the discs are not disturbed (by a bar or mergers, for example). Conversely, there is no clear correlation between $\nabla_{(\mathrm{O} / \mathrm{H})}$ and $\mathrm{R}_{\text {eff }}^{\mathrm{T}}$ or $\mathrm{R}_{\text {eff }}^{\text {star }}$.

By using the $\mathrm{D} / \mathrm{T}$ ratio as an indicative of galaxy morphology, we find a trend for discs in dispersion-dominated galaxies $(\mathrm{D} / \mathrm{T}<0.3)$ to show a clear trend between $\nabla_{(\mathrm{O} / \mathrm{H})}$ and $\mathrm{R}_{\text {eff }}^{\text {gas }}$. As can be seen from the right panel of Fig. 4 , the smaller the galaxy, the steeper negative $\nabla_{(\mathrm{O} / \mathrm{H})}$ it has. Disky galaxies $(\mathrm{D} / \mathrm{T}>0.3)$ show a shallower relation. This suggests that the latter have been able to enrich more efficiently the ISM while the formers have less evolved disc components.

\section{WHAT PHYSICS DETERMINES METALLICITY GRADIENTS?}

To explore how the $\nabla_{(\mathrm{O} / \mathrm{H})}$ are determined in the EAGLE discs, we analyse possible dependences of $\nabla_{(\mathrm{O} / \mathrm{H})}$ on other galaxy properties as a function of stellar mass. For this purpose, the original sample is divided into a high and a low stellar-mass subsamples, adopting $\mathrm{M}_{\text {star }}=10^{10.5} \mathrm{M}_{\odot}$ as a threshold. Within each mass subsample, discs are grouped according to the disc $\nabla_{(\mathrm{O} / \mathrm{H})}$ (i.e. negative or positive values). We find a slightly larger fraction of positive $\nabla_{(\mathrm{O} / \mathrm{H})}$ in 

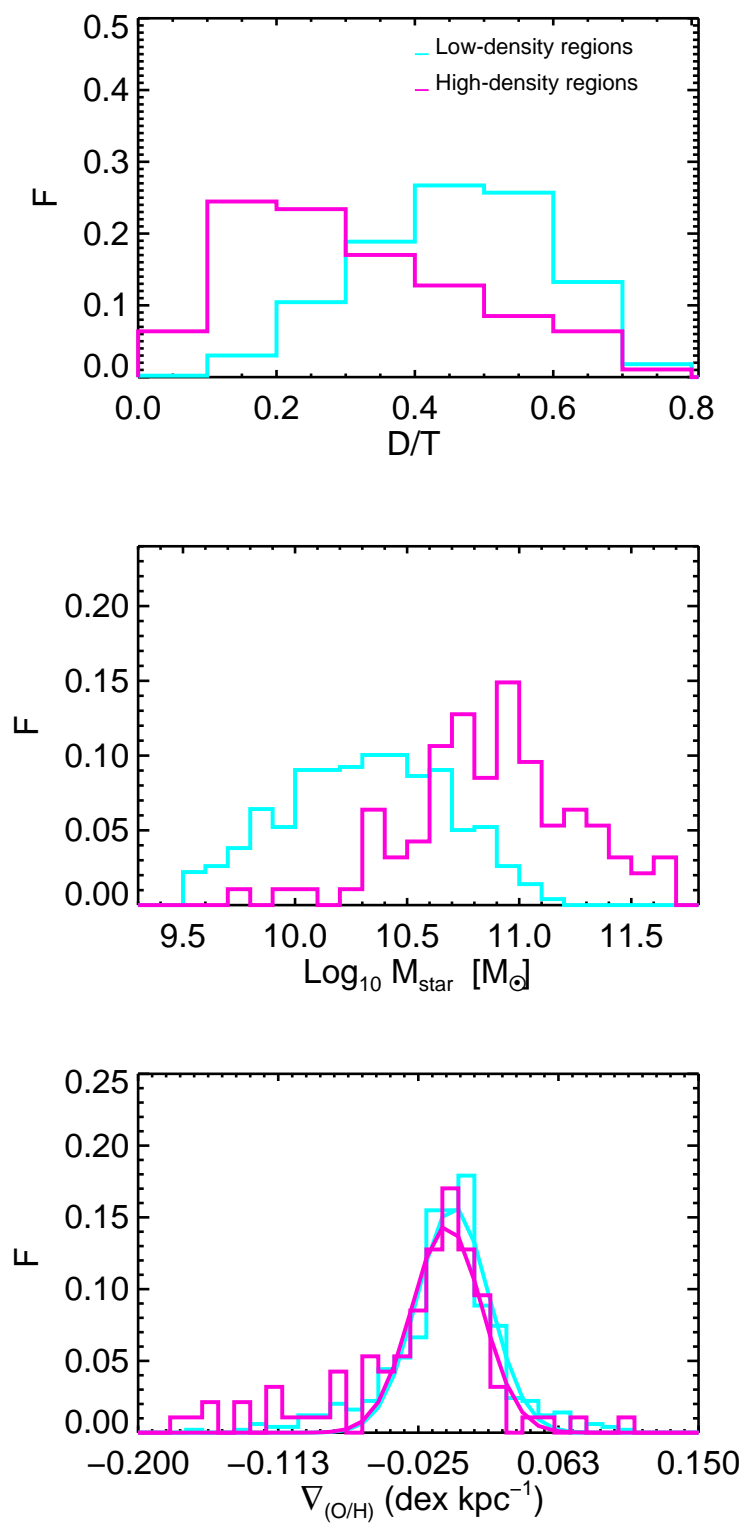

Figure 2. Distributions of $\mathrm{D} / \mathrm{T}$ ratios (upper), stellar masses (middle) and metallicity slopes, $\nabla_{(\mathrm{O} / \mathrm{H})}$, (lower) for the discs of the EAGLE galaxies in the two defined environments: low-density $\left(\mathrm{N}_{\mathrm{CC}} \leq 2\right.$; cyan line), and high-density $\left(\mathrm{N}_{\mathrm{CC}}>2\right.$; magenta lines $)$ regions. In the lower panel, the best Gaussian fits to the both simulated distributions are also shown.

the low-mass subsample, $0.43 \pm 0.06$, than in the high-mass one, $0.33 \pm 0.06$ (bootstrap errors are given).

In Fig. 5, the distributions of $\mathrm{D} / \mathrm{T}$ ratios, $\mathrm{R}_{\text {eff }}^{\text {star }}$, SFRs and sSFRs for discs with positive (magenta lines) and negative (blue lines) $\nabla_{(\mathrm{O} / \mathrm{H})}$ in the low (solid lines) and high (dashed lines) mass subsamples are shown. As can be seen from the first panel, there is a larger fraction of high mass galaxies with $\mathrm{D} / \mathrm{T}<0.3$ while low stellar-mass galaxies show a clear peak at $\mathrm{D} / \mathrm{T} \sim 0.5$. The $\mathrm{D} / \mathrm{T}$ distribution of massive galaxies is more uniform.

The second panel of Fig. 5 shows the distributions of $R_{\mathrm{eff}}^{\text {stars }}$ which are consistent with more massive galaxies having

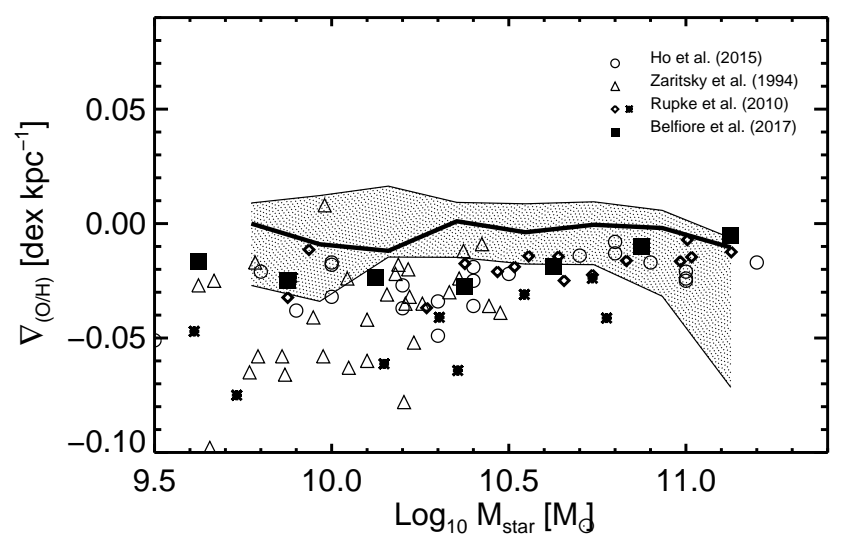

Figure 3. Median oxygen abundance slopes for the star-forming gaseous disc components as a function of stellar mass of the selected EAGLE galaxies (black line). The shaded areas are defined by the first and third quartiles. For comparison, observational results from Zaritsky et al. (1994, open triangles), Rupke et al. (2010, open diamonds), Ho et al. (2015, open circles) and Belfiore et al. (2017, filled squares) are included.

slightly larger discs $R_{\text {eff }}^{\text {star }}$ (Furlong et al. 2015). However, no clear differences in the size distributions are found for galaxies with positive or negative $\nabla_{(\mathrm{O} / \mathrm{H})}$ in a given stellar-mass interval (according to a KS test). The third panel of Fig. 5 shows the expected trend for massive galaxies to have higher SFRs. The distributions of massive galaxies with positive $\nabla_{(\mathrm{O} / \mathrm{H})}$ are slightly displaced towards lower SFR compare to those with negative $\nabla_{(\mathrm{O} / \mathrm{H})}$. Smaller galaxies with positive and negative $\nabla_{(\mathrm{O} / \mathrm{H})}$ show no differences in the sSFR distributions as can be seen in the fourth panel.

The KS tests performed over these distributions provide no statistical differences, with only a weak signal for massive galaxies with positive $\nabla_{(\mathrm{O} / \mathrm{H})}$ to be less active. Recall that, as shown in Fig.4, there is a strong dependence of $\nabla_{(\mathrm{O} / \mathrm{H})}$ on $R_{\text {eff }}^{\text {gas }}$

\subsection{Disc assembly and merger history}

In order to get further insight in the origin of the characteristics of the abundance profiles, in this section, we explore a set of properties related to the history of disc assembly the EAGLE discs.

First, we calculate the cumulative fraction of the stellar mass formed as a function of stellar age for discs in each of the subsamples used in the previous section. We define $T^{30}$ as the lookback time (Gyr) when the last 30 per cent of the stellar populations was formed in each disc. This parameter aims at capturing the recent star formation activity that might be related with injection of chemical elements and/or the triggering of outflows, which could affect the chemical abundances profiles on the discs in the recent past.

Figure 6 shows the $T^{30}$ distributions for the four analysed subsamples. As can be seen, high mass galaxies formed their 30 per cent youngest stellar population earlier than low mass galaxies by $\sim 1-1.5$ Gyr. Within each mass subsample, discs with negative metallicity slopes have typically 

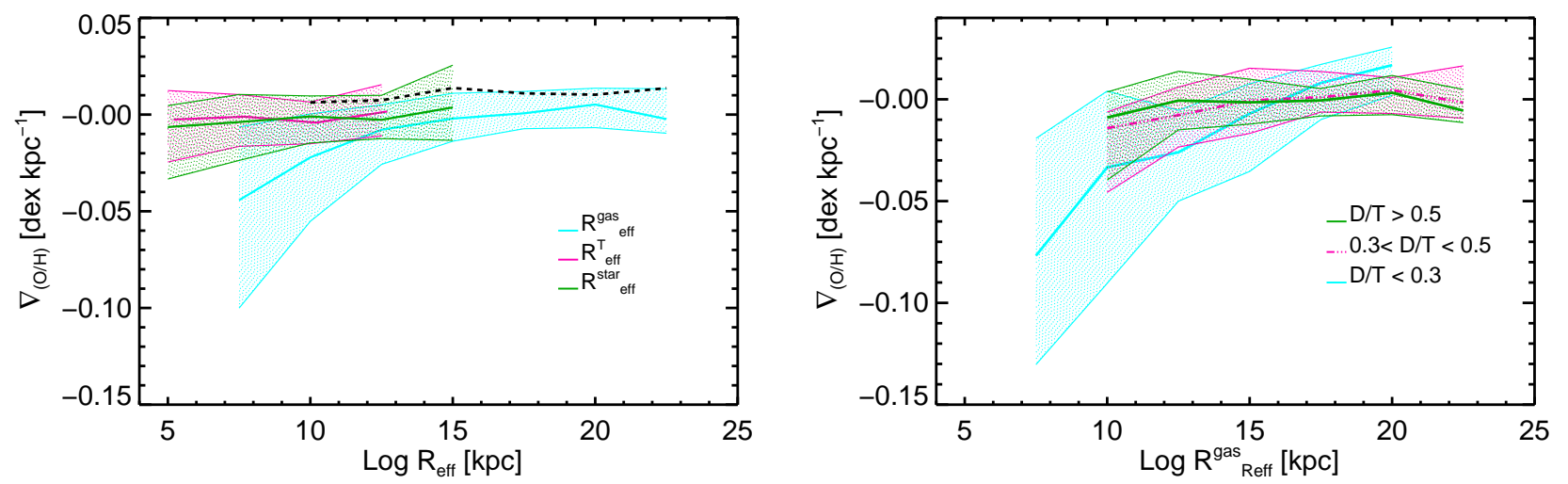

Figure 4. Median oxygen abundance slopes for the star-forming gaseous disc components as a function of disc size of the selected EAGLE galaxies. Left panel: Median values are shown as a function of stellar $\left(\mathrm{R}_{\mathrm{eff}}^{\text {star }}\right)$ and gas $\left(\mathrm{R}_{\mathrm{eff}}^{\text {gas }}\right)$ half-mass radius (green line and cyan lines, respectively) and the galaxy stellar half-mass radius ( $\mathrm{R}_{\text {eff }}^{\mathrm{T}}$; magenta line). The shaded areas are defined by the first and third quartiles. Additionally, the relation for galaxies with positive metallicity gradients in the disc components is shown (black dashed line). Right panel: Median oxygen abundance slopes as a function of $\mathrm{R}_{\text {eff }}^{\text {gas }}$ for galaxies grouped according to $\mathrm{D} / \mathrm{T}$ ratios.

younger stellar populations than those with positive metallicity slopes. The differences are statistically significant according to a KS test, although they are larger for the low mass galaxies (KS tests: $p=0.09$ and $p=0.19$, with a similar deviation $\approx 0.30$, for the low and high mass subsamples, respectively). Hence, positive metallicity gradients are associated with discs that have more active star formation $\approx 1.5$ Gyr ago, on average, while negative metallicity slopes tend to be related with galaxies that have more recent star formation activity.

The stellar and AGN feedback play an important role in the regulation of the star formation activity by heating the dense gas clouds, quenching star formation. Additionally, metal mass-loaded outflows can be triggered, removing material from the galaxies that can modify the chemical patterns. Considering that oxygen is released on short time-scales $\left(\sim 10^{7} \mathrm{yr}\right)$, significant fractions of the $\alpha$-elements could have been transported outwards by galactic winds (Oppenheimer et al. 2017; Machado et al. 2018). If the feedback mechanisms eject material preferentially from the inner regions of galaxies then, the mean central oxygen abundances should be lower in discs with positive $\nabla_{(\mathrm{O} / \mathrm{H})}$ than those with negative $\nabla_{(\mathrm{O} / \mathrm{H})}$. Even more, if the ejection of enriched material in the central regions were the dominant mechanism, we would expect the outer regions of the discs to have the same level of enrichment regardless of $\nabla_{(\mathrm{O} / \mathrm{H})}$ (i.e. positive or negative $\left.\nabla_{(\mathrm{O} / \mathrm{H})}\right)$. Secular evolution will also work in the same direction by driving inwards less-enriched gas from the outer regions of the discs, diluting the central metallicities (e.g. Di Matteo et al. 2009; Perez et al. 2011; Sillero et al. 2017). Conversely, similar central abundances and different levels of enrichment in the outer parts would imply different gas accretion histories in the outskirts, with significant contribution of enriched material in those discs with positive slopes at $z=0$.

Therefore, to assess if the metallicity gradients are consistent with a decrease of the central metallicity (independently of the physical mechanisms responsible for such effect), in the upper panel of Fig. 7, we show the central oxygen abundance for discs with positive (magenta, dashed lines) and negative (blue, solid lines) $\nabla_{(\mathrm{O} / \mathrm{H})}$. The lower panel shows the oxygen abundance at twice the stellar disc effective radius. It is clear that the metallicities of the gas in the disc outskirts are similar for both types of systems. However, discs with positive abundance gradients have systematically lower level of enrichment in the central regions. As mentioned above, the opposite trend would be expected if positive gradients were built mainly as a result of significant accretion of enriched material in the disc outskirts. This suggests the action of either secular evolution and/or the stellar and AGN feedback to remove enriched gas from the central regions. The analysis of the EAGLE set of simulations with the same initial conditions but with different SN feedback energy yield a trend to have more positive slopes for higher SN energy released per event in low mass galaxies (see Appendix A.2).

For the purpose of estimating the possible contribution of re-accreted material to the discs that was first directly heated by feedback mechanisms, we search for the disc stars formed from gas that was previously heated up by more than $10^{7} \mathrm{~K}$ (see Section 2). In Fig. 8, we show the fractions of disc stars formed from heated gas particles, located in the inner and outer regions of the discs as a function of $\nabla_{(\mathrm{O} / \mathrm{H})}$. The inner and outer regions have been defined by using the $R_{\text {eff }}^{\text {star }}$ as a threshold. As can be seen from this figure, the contribution of re-heated gas is larger in the central regions than in the outskirts of discs. There is a slight trend to have large fraction in the central regions of discs with negative metallicity gradients. This heated and enriched gas contributes to reinforce the formation of negative metallicity gradients. In the outer regions, the fractions are less than five per cent. Hence, the re-accretion of enriched material ejected by feedback mechanisms is not contributing significantly in the outer parts of the discs, supporting the conclusions drawn from Fig. 7.

The gas depletion times $\left(\tau_{\text {Dep }}=\mathrm{M}(\mathrm{HI})+\mathrm{M}(\mathrm{H} 2) / \mathrm{SFR}\right)$ provide an estimate of the importance of the star formation activity in relation to the gas availability of the system. To estimate them, we use the $\mathrm{HI}$ and $\mathrm{H} 2$ masses $(\mathrm{M}(\mathrm{HI})+\mathrm{M}(\mathrm{H} 2))$ calculated by Lagos et al. (2015) by ap- 

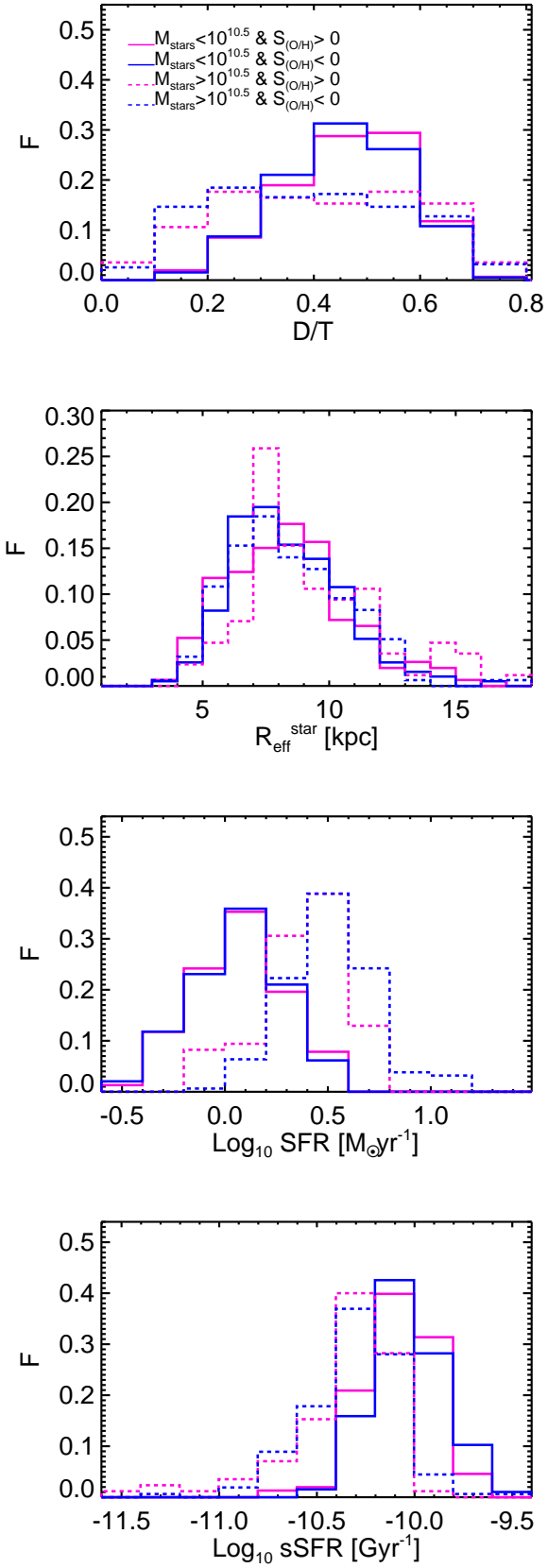

Figure 5. Stellar mass-weighted distributions of D/T ratios (first panel), $R_{\text {eff }}^{\text {stars }}$ (second panel), SFR (third panel) and sSFR (fourth panel) for $M_{\text {star }}<10^{10.5} \mathrm{M}_{\odot}$ (solid lines) and $M_{\text {star }}>10^{10.5} \mathrm{M}_{\odot}$ (dotted lines) galaxies with positive (magenta lines) and negative (blue lines) oxygen abundance gradients.

plying the model of Krumholz $(2013)^{4}$. As can be seen from Fig. 9, massive galaxies have shorter neutral gas depletion times than lower stellar mass galaxies as a result of their higher gas surface densities. Interestingly, within each mass interval, galaxies with negative $\nabla_{(\mathrm{O} / \mathrm{H})}$ tend to have longer depletion times (KS tests: $0.43, p=0.18$ and $0.9, p=0.22$

\footnotetext{
${ }^{4}$ Similar relative trends are found if the star-forming gas is used to estimate the depletion times.
}

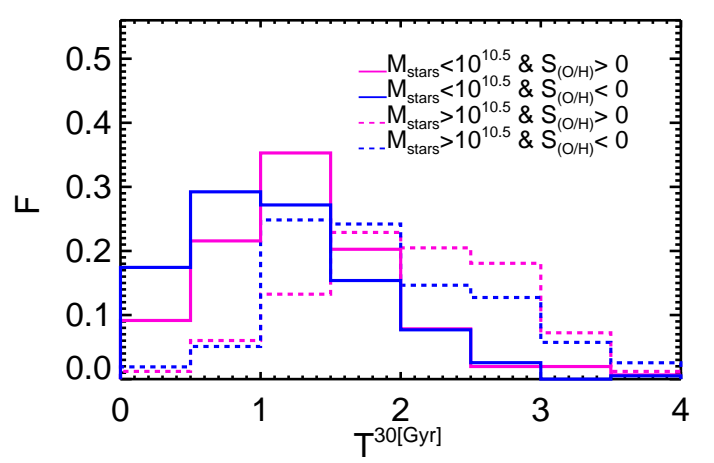

Figure 6. Distributions of lookback times corresponding to the formation of the youngest 30 per cent of stars for high (dashed lines) and low (solid lines) stellar mass galaxies with discs exhibiting positive (magenta lines) and negative (blue lines) gaseous oxygen abundance slopes. Discs with negative oxygen gradients tend to have more recent star formation activity.
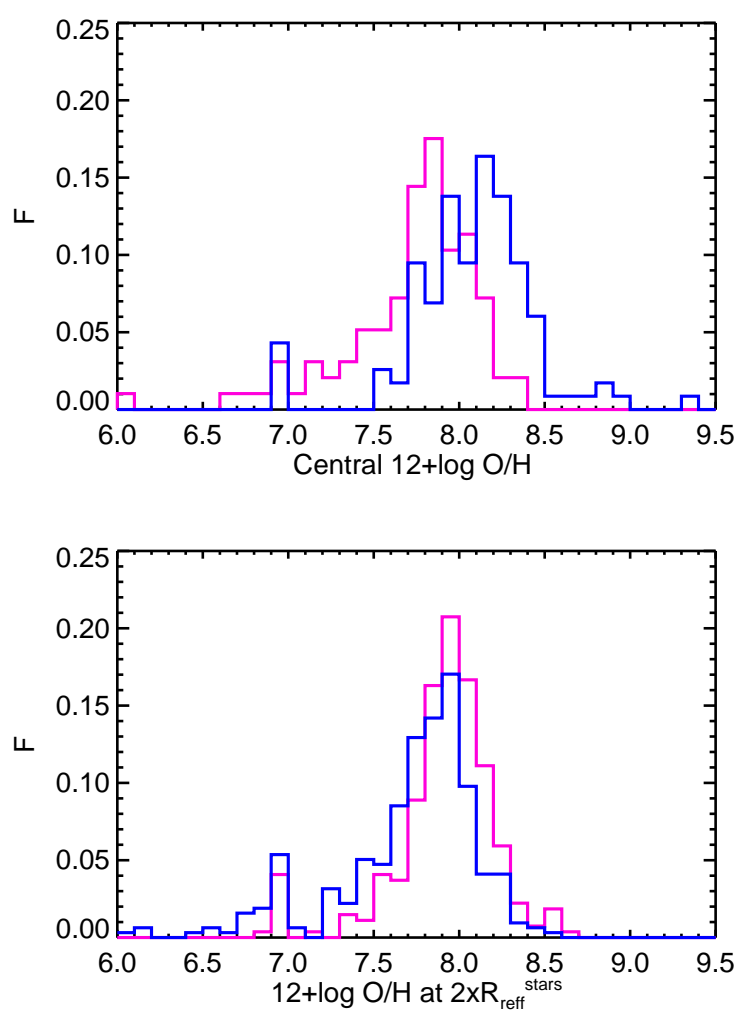

Figure 7. Central gaseous oxygen abundance for discs with positive (magenta, solid lines) and negative (blue dashed lines) metallicity profiles (upper panel) and the gaseous oxygen abundance at twice the stellar half-mass radius of the discs (lower panel).

for the low and high mass subsamples, respectively). This is consistent with the fact that these galaxies have been able to extend their star formation activity to more recent times. We note that the differences are stronger for more massive galaxies.

From the previous analysis, we find that discs with neg- 


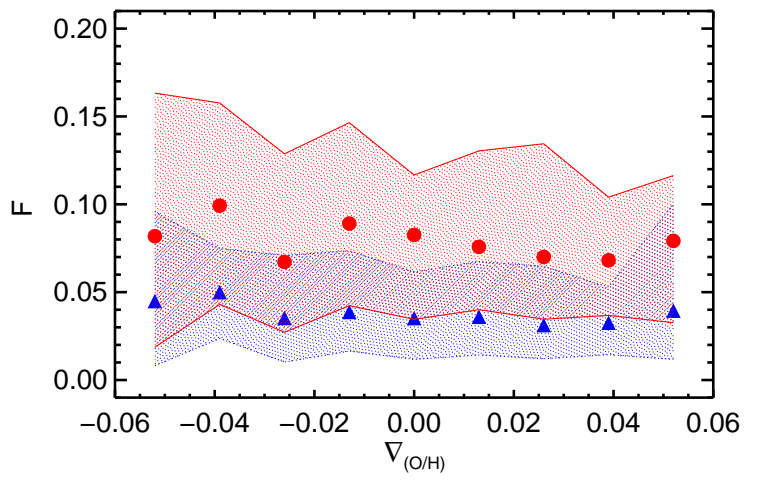

Figure 8. Median fraction of the stellar mass that was formed from directly heated and enriched gas that, later on, was reaccreted to contribute to the formation of the inner (red, circles) and outer (blue, triangles) regions of the discs as a function of the oxygen abundance gradients. The shaded areas encompasses the 25 and 75 percentile.

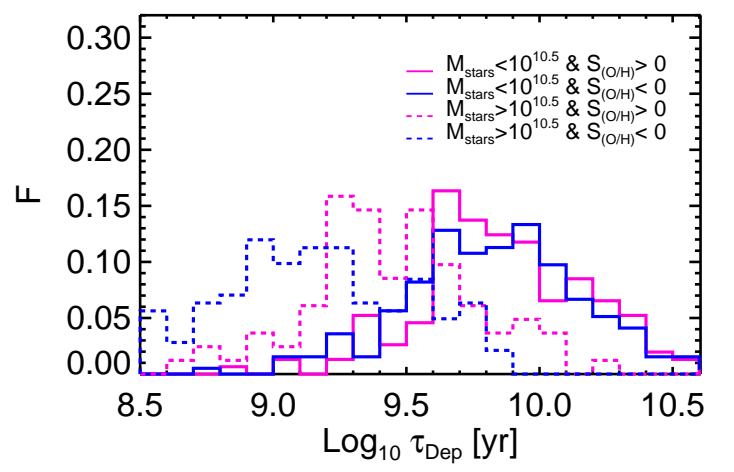

Figure 9. Distributions of the depletion times estimated using the HI and H2 gas mass estimated by Lagos et al. (2015) for high (dashed lines) and low (solid lines) stellar mass galaxies with discs exhibiting positive (magenta lines) and negative (blue lines) oxygen abundance slopes.

ative $\nabla_{(\mathrm{O} / \mathrm{H})}$ tend to have formed stars more actively in the recent past, have slightly higher contribution of stars formed from heated gas by feedback in the central disc regions and longer depletion times than those with positive $\nabla_{(\mathrm{O} / \mathrm{H})}$. The comparison of the inner and outer levels of enrichments of discs with positive and negative $\nabla_{(\mathrm{O} / \mathrm{H})}$ is consistent with the action of mechanisms that diluted the central abundances in discs with positive $\nabla_{(\mathrm{O} / \mathrm{H})}$.

As clearly shown in Fig. 3, the EAGLE discs have median negative gradients that do not vary significantly as a function of stellar mass when all galaxies in the analysed sample are considered. However, they are shallower than observed. To understand the origin of this overall weak median metallicity gradient (see the lower panel of Fig. 2), in Fig. 10 we show the ratio of the star-forming gas surface density $\left(\Sigma_{\text {SFgas }}\right)$ to the stellar surface density of stars younger than 2 Gyrs $\left(\Sigma_{\text {star }}\right)$ for discs with high and low stellar mass and within each subsample, for those with negative and positive metallicity gradients, as a function of $r / R_{\mathrm{eff}}^{\text {star }}$. As can be seen

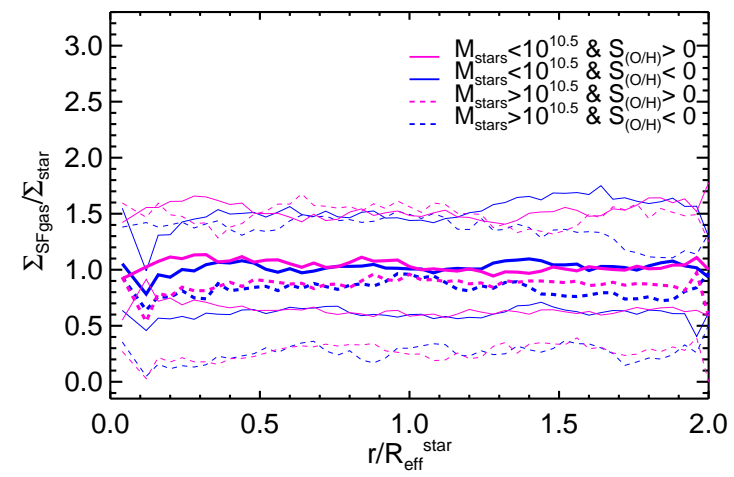

Figure 10. Median ratio between the gas surface density $\Sigma_{\text {SFgas }}$ and the young stellar surface density $\Sigma_{\text {star }}$ as a function of $r / R_{\text {eff }}^{\text {star }}$ for EAGLE discs with positive (magenta lines) and negative (blue lines) gaseous oxygen abundance slopes. The profiles are shown for high (dashed lines) and low (solid lines) mass galaxies. The thin lines denote the first and third quartiles.

the median ratios are close to unity as a function of radius, implying similarly high and similar efficiency to transform the available gas into stars regardless of the location of the star-forming regions in the discs. This trend suggests that the oxygen content in the ISM is built up quickly, resulting in flat oxygen profiles (e.g. Pilkington et al. 2012; Mollá et al. 2017). This efficient star formation activity provides an interpretation to the overall weak (negative/positive) metallicity gradients found in the EAGLE discs.

It is important to note that there is a large spread in the star forming gas-to-stellar surface density ratios that accounts for a wide variety of individual behaviours determined by each evolutionary history, as can be seen from Fig. 10 (thin lines denote the first and third quartiles).

\subsection{The role of mergers and the environment}

For the selected EAGLE galaxies, we estimate the mass and time of merger events with relative stellar mass ratios larger than 1:10. In Fig. 11, we show $\nabla_{(\mathrm{O} / \mathrm{H})}$ as a function of stellar masses for galaxies that had merger events and those that did not (hereafter, non-merger galaxies). Galaxies with quiet assembly histories show a clearer trend with stellar mass. This trend is robust against numerical resolution as can be seen from Appendix A.1, where we show results for the highresolution Recal-L025N0752 simulation.

In Fig. 11, we also display the relation for those galaxies with $\mathrm{D} / \mathrm{T}>0.5$ and gas fraction larger than 15 per cent (dashed line). These galaxies follow the relation for nonmerger galaxies and are more comparable to spiral galaxies for which observational results are commonly reported (Ho et al. 2015, and references therein). However, we note that the stellar mass range analysed in observations extends to lower stellar masses which are not well-resolved in the Ref-L100N1504 simulation. To extend the analysis to lower masses, a similar study is performed on the RecalL025N0752, finding that the simulated trend continues down to $\sim 10^{9.2} \mathrm{M}_{\odot}$ (see Appendix A.1). If a stellar mass threshold of $10^{10} \mathrm{M}_{\odot}$ is assumed, we estimate median $\nabla_{(\mathrm{O} / \mathrm{H})}$ of $-0.010 \pm 0.002$ and $-0.015 \pm 0.005$ for the high and low stellar- 
mass subsamples, respectively. The change of the slopes is consistent with observational results in the sense that smaller galaxies tend to have more negative gradients, on average, although the difference in simulations is marginal.

For comparison with other simulations, in Fig. 11, we have also included the $\nabla_{(\mathrm{O} / \mathrm{H})}$ reported by Tissera et al. (2016) and Ma et al. (2017). Both works find steeper negative metallicity gradients. The main differences between these works originate in the different subgrid physics that affects the regulation of the star formation activity and the mixing of chemical elements (see Introduction).

In Fig. 12 we show $\nabla_{(\mathrm{O} / \mathrm{H})}$ as a function of the environment. The value $\mathrm{N}_{\mathrm{CC}}=0$ corresponds to isolated galaxies within the $500 \mathrm{kpc}$ cube volume, according to the adopted criteria discussed in Section 2. As can be seen from this figure, there are no clear variations with local environment. The small decrease towards more negative metallicity gradients for larger groups is produced by a larger contribution of more massive dispersion-dominated galaxies located in denser environments. These galaxies have small-size disc components (Fig.4). The variations between the median gradients as a function of environment are within the standard deviations.

The median values for galaxies with $D / T>0.5$ are included in Fig. 12 (green triangles). They also show no trend with $\mathrm{N}_{\mathrm{CC}}$. To better compare with the recent observational findings by Sánchez-Menguiano et al. (2018), the normalised metallicity gradients are additionally shown (open blue squares). Our results agree with observations that reports no clear effects associated to variation in the global environment (red solid circles). However, as mentioned before, a larger galaxy sample covering the same mass range in different environments is required to improve this analysis.

\subsection{Normalised oxygen gradients}

Observational results suggest the existence of a characteristic metallicity gradient when the abundance profiles are normalised by a scale-length related to the size of the galaxies (e.g. Sánchez et al. 2013). Because the size of galaxies correlates with stellar mass, the renormalisation of the metallicity profiles by the effective radius (dex reff ${ }_{\text {eff }}^{-1}$ removes such a trend.

As discussed above, on the one hand, $\nabla_{(\mathrm{O} / \mathrm{H})}$ shows no clear trend with stellar mass, except for non-merger galaxies that have weaker $\nabla_{(\mathrm{O} / \mathrm{H})}$ for more massive galaxies. On the other hand, the relation between galaxy size and stellar mass is well-reproduced by the EAGLE galaxies (Schaye et al. 2015). The stellar disc scale-lengths $R_{\text {eff }}^{\text {star }}$ show a correlation with stellar mass albeit slightly weaker (Tissera et al. 2018, in preparation) than that reported for CALIFA disc galaxies by Sánchez-Blázquez et al. (2014).

We estimate the normalised $\nabla_{(\mathrm{O} / \mathrm{H})}$ within the same radial range $[0.5,2] R_{\text {eff }}^{\text {star }}$ used in the previous section. The normalisation has been also done by using $R_{\text {eff }}^{\mathrm{T}}$. Fig. 13 shows the distributions of the normalised $\nabla_{(\mathrm{O} / \mathrm{H})}$ for the two defined environments (we applied the same criteria explained in Section 2). As can be seen from the figure, the normalised oxygen gradients are in general shallower than those reported for the CALIFA galaxies by Sánchez et al. (2013). In both panels, we include the best Gaussian fits to the $\nabla_{(\mathrm{O} / \mathrm{H})}$ distri-
Table 2. Parameters of the Gaussian fits to the normalised $\nabla_{(\mathrm{O} / \mathrm{H})}$ distributions of galaxy discs in low $\left(N_{\mathrm{CC}} \leq 2\right)$ and high $N_{\text {CC }}>2$ density regions.

\begin{tabular}{lccccc}
\hline$\nabla_{(\mathrm{O} / \mathrm{H})}$ & $N_{\mathrm{CC}}$ & Height & $\nabla_{(\mathrm{O} / \mathrm{H})}$ central & $\sigma$ & $\chi^{2}$ \\
\hline \multirow{3}{*}{$\operatorname{dex} / \mathrm{R}_{\text {eff }}^{\text {star }}$} & $N_{\mathrm{CC}}>2$ & 0.10 & -0.07 & 0.19 & 0.0004 \\
& $N_{\mathrm{CC}} \leq 2$ & 0.11 & -0.03 & 0.17 & 0.0004 \\
& All & 0.11 & -0.03 & 0.18 & 0.0001 \\
$\operatorname{dex} / \mathrm{R}_{\text {eff }}^{\mathrm{T}}$ & $N_{\mathrm{CC}}>2$ & 0.12 & -0.06 & 0.14 & 0.0003 \\
& $N_{\mathrm{CC}} \leq 2$ & 0.13 & -0.02 & 0.14 & 0.0001 \\
& All & 0.13 & -0.03 & 0.14 & 0.0001 \\
\hline
\end{tabular}

butions in low-and-high density regions. As can be seen, distributions are slightly displaced towards more positive values for discs in low-density regions (see Table 2 for a summary of the fitting parameters). The gaussian fits to the total distributions yield centred values at -0.03 dexR $\mathrm{e}_{\mathrm{eff}}^{-1}$ for both of them. However the standard deviations are narrower when the abundance profiles are normalised by $\mathrm{R}_{\mathrm{eff}}^{\mathrm{T}}$.

In Fig. 14, the normalised gradients as a function of stellar mass are displayed. As can be seen from this figure there are no clear trend with stellar mass in agreement with observations. The scatter of $\nabla_{(\mathrm{O} / \mathrm{H})}$ normalised by $\mathrm{R}_{\text {eff }}^{\mathrm{T}}$ is significant smaller as mentioned before. This is expected considering that there is a clearer correlation between this scale-length and galaxy stellar mass. Similar behaviours are found for non-merger galaxies. In agreement with observational results, the relation between $\nabla_{(\mathrm{O} / \mathrm{H})}$ and stellar mass is no longer present in the normalised gradients.

\section{CONCLUSIONS}

We analysed the oxygen abundance gradients of the starforming gas in discs of 592 central galaxies, identified in the Ref-L100N1504 simulation of the EAGLE project. We studied them as a function of stellar masses, recent merger history, gas richness and global environment. We also analysed the high resolution Recal-L025N0752 and a suite of smaller volume runs with different subgrid physics (see Appendix).

Our main results can be summarised as follows:

- The median oxygen gradients of the EAGLE discs are negative, $-0.011 \pm 0.002 \mathrm{dex} \mathrm{kpc}^{-1}$, albeit shallower than observed. The negative slopes are a consequence of the global inside-out disc assembly. We find no clear trend of $\nabla_{(\mathrm{O} / \mathrm{H})}$ with stellar mass (Fig. 3).

- The $\nabla_{(\mathrm{O} / \mathrm{H})}$ is found to correlate with the scale-length of the gas discs so that shallower slopes are measured in larger discs (Fig. 4). Small gas discs show a larger variety of $\nabla_{(\mathrm{O} / \mathrm{H})}$ with most disc negative gradients in dispersiondominated galaxies (Rosito et al., in preparation). The correlation is found to be mainly determined by discs with negative $\nabla_{(\mathrm{O} / \mathrm{H})}$ while discs with positive $\nabla_{(\mathrm{O} / \mathrm{H})}$ show no trend with gas scale-length.

- There is a significant fraction of galaxies with positive $\operatorname{disc} \nabla_{(\mathrm{O} / \mathrm{H})}(\sim 40$ per cent $)$. These galaxies show important star formation activity $\approx 1.5$ Gyr before those with negative gradients at a similar stellar mass (Fig. 6). Analysing the gas abundances of the inner and outer parts of discs, we find evidence suggesting the action of the stellar/AGN feedbacks that preferentially removes metal-rich gas from the central 


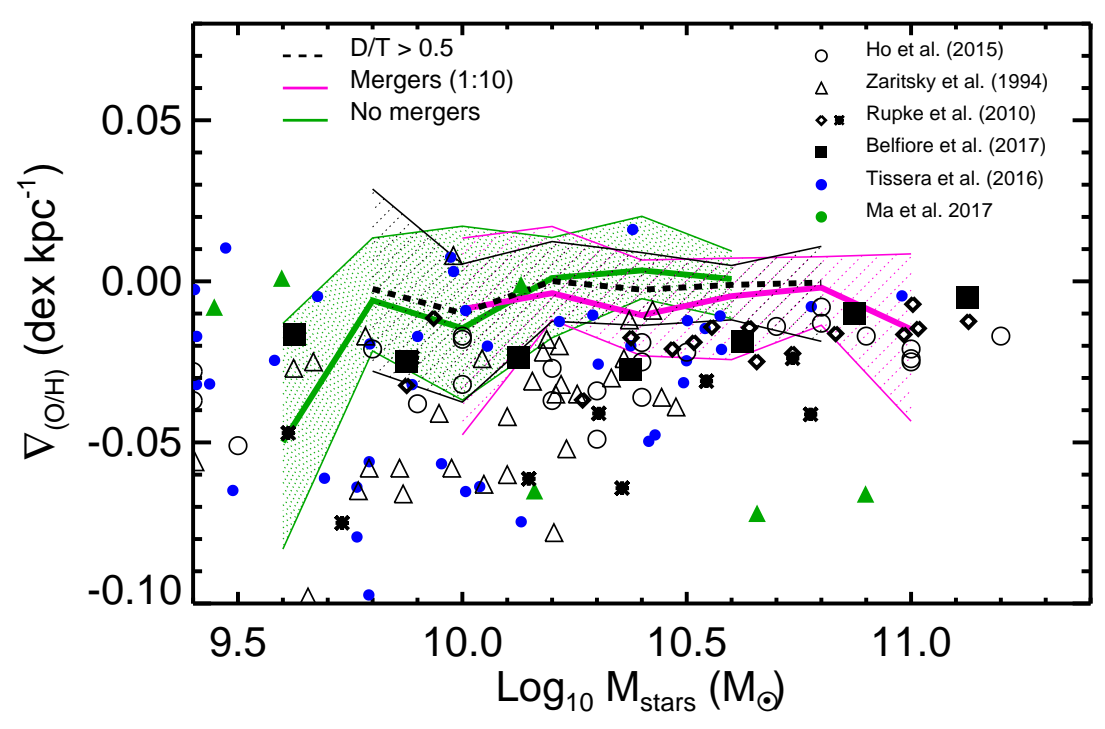

Figure 11. Median abundance gradients estimated for the star-forming disc components as a function of the stellar mass of the EAGLE galaxies. Galaxies which experienced no mergers determine a correlation between the oxygen abundance gradients and the stellar mass (green lines). Those which have had a merger (more massive than 1:10) are displayed separately and determine a flat relation (magenta line). The relation determined for galaxies with $\mathrm{D} / \mathrm{T}>0.5$ and total gas fraction larger than 0.15 is also shown for comparison (black line). The shaded areas are defined by the first and third quartiles. For comparison observational results from Zaritsky et al. (1994, open triangles), Rupke et al. (2010, black diamonds), Ho et al. (2015, open circles) and Belfiore et al. (2017, fillerd squares) are included. We also include the simulated results by Tissera et al. (2016, blue solid circles) and Ma et al. (2017, green filled triangles) for metallicity gradients of discs simulated with different numerical codes and subgrid physics.

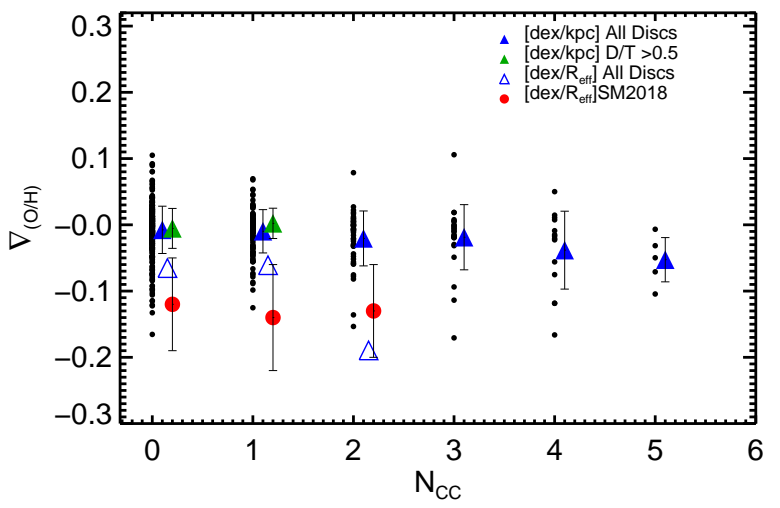

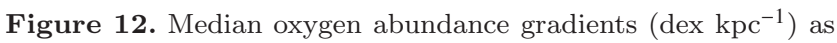
a function of $N_{\mathrm{CC}}$ for all analysed discs (blue triangles), for discs with $\mathrm{D} / \mathrm{T}>0.5$ (green triangles). Median normalised gradients $\left(\mathrm{dex} / R_{\mathrm{eff}}^{\text {star }}\right)$ are also included (open blue triangles). Individual gradients for the simulated discs are shown (small black circles). For comparison, the mean normalised metallicity gradients reported by Sánchez-Menguiano et al. (2018) are also included (red filled circles; MS2018).

regions (Fig. 7). Secular evolution may also contribute by diluting the central abundances. There is a weak trend for galaxies with positive $\nabla_{(\mathrm{O} / \mathrm{H})}$ to prefer low-density regions (Fig. 2 and Fig. 13).

- The contribution to the formation of the discs and the abundance gradients of material previously heated up by
stellar/AGN feedback, and later on re-accreted, is small in the analysed discs (below 10 per cent, on average). However, we detect slightly larger contributions of re-accreted material in the central regions of discs with negative $\nabla_{(\mathrm{O} / \mathrm{H})}$ than in those with positive ones. In the outer regions of discs, this contribution is smaller (about 5 per cent on average) and similar for all discs, regardless of their oxygen slopes (Fig. 8).

- The weak metallicity gradients measured in the EAGLE discs can be ascribed to the overall high star formation efficiency detected in the discs (regardles of radius (Fig. 10). The gas with physical conditions suitable for forming stars is transformed efficiently along the discs. Therefore, oxygen profiles converge quickly to a weak metallicity gradient, on average. However, there is significant dispersion that reflects the large variation in the star formation histories of the discs.

- Galaxies with quiet merger histories show a positive trend between $\nabla_{(\mathrm{O} / \mathrm{H})}$ and stellar mass so that lower mass galaxies tend to have steeper metallicity gradients, on average (Fig. 11). Non-merger galaxies tend to be rotationaldominated $(\mathrm{D} / \mathrm{T}>0.5)$. However, at low stellar masses, there is a significant contribution of intermediate morphologies $(0.3<\mathrm{D} / \mathrm{T}<0.5)$. These galaxies tend to be located in low-density environments. The normalisation of the oxygen profiles by the disc scale-length tends to remove the trend, in agreement with observations (Fig. 14). These findings suggest that the large variety of gas-phase oxygen gradients at a given stellar mass reported by observations might originate in the fact that galaxies with different assembly histories are considered together.

- No clear differences in the gas disc $\nabla_{(\mathrm{O} / \mathrm{H})}$ are found be- 

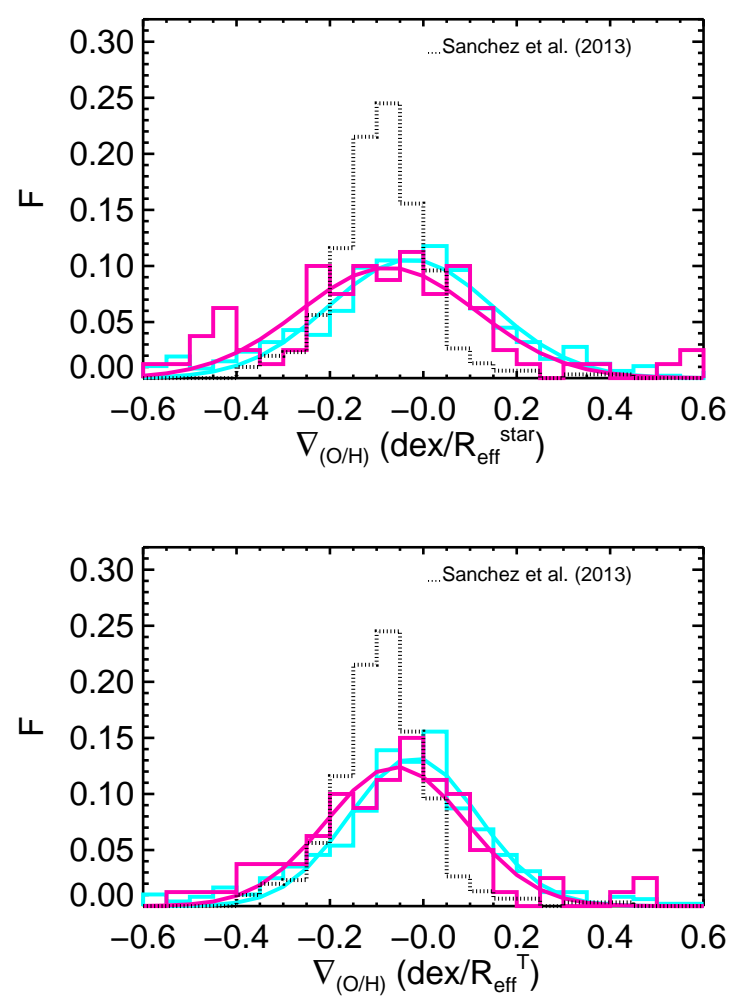

Figure 13. Distribution of the normalised metallicity gradients of the star-forming gas in the analysed EAGLE discs by using the $\mathrm{R}_{\text {eff }}^{\text {star }}$ (upper panel) and $\mathrm{R}_{\text {eff }}^{\mathrm{T}}$ (lower panel), in the two defined environments (low density $\left(N_{\mathrm{CC}} \leq 2\right)$ ) cyan lines and high density $\left(N_{\mathrm{CC}}>2\right.$ : magenta lines) as given in Fig.2. The two solid lines represent the best gaussian fits to each distribution. For comparison, the observational distribution of normalised slopes reported by Sánchez et al. (2013) is included (black, dashed line).

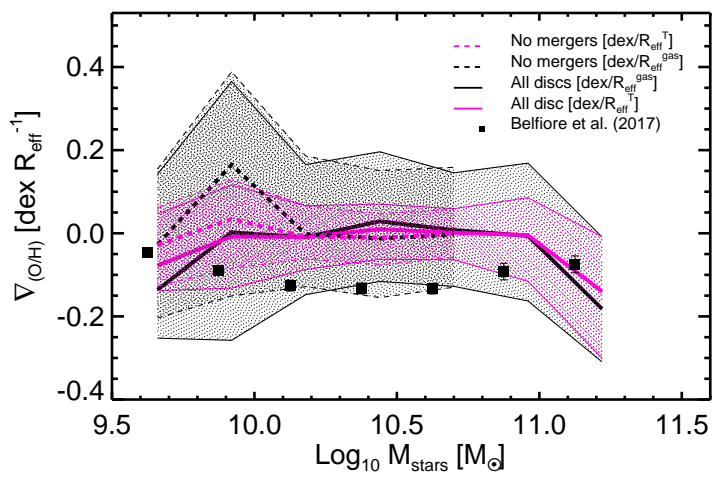

Figure 14. Median oxygen gradients of the star-forming gas in discs as a function of stellar mass normalised by $\mathrm{R}_{\mathrm{eff}}^{\text {star }}$ (black lines) and $R_{\text {eff }}^{T}$ (magenta lines). Similar relations for galaxies with no mergers are also included (dashed lines). For comparison, the observational results from Belfiore et al. (2017) are included (black squares). tween galaxies located in groups and in isolation, in agreement with recent observational results (Fig. 12). However, we note that the analysed disc sample does not cover the same mass range in all environments. Hence, a larger and higher resolution galaxy sample is needed to analyse this effect in more detail.

\section{ACKNOWLEDGMENTS}

RAC is a Royal Society University Research Fellow. CL is funded by a Discovery Early Career Researcher Award (DE150100618) and by the Australian Research Council Centre of Excellence for All Sky Astrophysics in 3 Dimensions (ASTRO 3D), through project number CE170100013. MS acknowledges the VENI grant 639.041.74. PBT acknowledges partial funding by Fondecyt Regular 2015 - 1150334. This project has received funding from the European Union Horizon 2020 Research and Innovation Programme under the Marie Sklodowska-Curie grant agreement No 734374.

This work used the DiRAC Data Centric system at Durham University, operated by the Institute for Computational Cosmology on behalf of the STFC DiRAC HPC Facility (www.dirac.ac.uk). This equipment was funded by BIS National E-infrastructure capital grant ST/K00042X/1, STFC capital grants ST/H008519/1 and ST/K00087X/1, STFC DiRAC Operations grant ST/K003267/1 and Durham University. DiRAC is part of the National EInfrastructure. We acknowledge PRACE for awarding us access to the Curie machine based in France at TGCC, CEA, Bruyeres-le-Chatel.

\section{REFERENCES}

Amorín R., Vílchez J. M., Hägele G. F., Firpo V., Pérez-Montero E., Papaderos P., 2012, ApJL, 754, L22

Artale M. C., et al., 2017, MNRAS, 470, 1771

Aumer M., White S. D. M., Naab T., Scannapieco C., 2013, MNRAS, 434, 3142

Barnes J. E., Hernquist L., 1996, ApJ, 471, 115

Belfiore F., et al., 2017, MNRAS, 469, 151

Bland-Hawthorn J., Freeman K. C., 2003, in E. Perez, R. M. Gonzalez Delgado, \& G. Tenorio-Tagle ed., Astronomical Society of the Pacific Conference Series Vol. 297, Star Formation Through Time. pp 457-+

Calura F., et al., 2012, MNRAS, 427, 1401

Carton D., et al., 2018, MNRAS,

Chabrier G., 2003, ApJ, 586, L133

Chiappini C., Matteucci F., Romano D., 2001, ApJ, 554, 1044

Cora S. A., 2006, MNRAS, 368, 1540

Crain R. A., et al., 2015, MNRAS, 450, 1937

Crain R. A., et al., 2017, MNRAS, 464, 4204

Dalla Vecchia C., Schaye J., 2012, MNRAS, 426, 140

De Lucia G., Tornatore L., Frenk C. S., Helmi A., Navarro J. F., White S. D. M., 2014, MNRAS, 445, 970

De Rossi M. E., Bower R. G., Font A. S., Schaye J., Theuns T., 2017, MNRAS, 472, 3354

Di Matteo P., Pipino A., Lehnert M. D., Combes F., Semelin B., 2009, A\&A, 499, 427

Dolag K., Borgani S., Murante G., Springel V., 2009, MNRAS, 399, 497

Dressler A., 1980, ApJ, 236, 351

Ellison S. L., Patton D. R., Simard L., McConnachie A. W., 2008, AJ, 135,1877

Fall S. M., Efstathiou G., 1980, MNRAS, 193, 189 
Ferland G. J., et al., 2013, Rev. Mexicana Astron. Astrofis., 49, 137

Furlong M., et al., 2015, MNRAS, 450, 4486

Genel S., et al., 2018, MNRAS, 474, 3976

Gibson B. K., Pilkington K., Brook C. B., Stinson G. S., Bailin J., 2013, A\&A, 554, A47

Haardt F., Madau P., 2001, in Neumann D. M., Tran J. T. V., eds, Clusters of Galaxies and the High Redshift Universe Observed in X-rays. (arXiv:astro-ph/0106018)

Hayden M. R., et al., 2015, ApJ, 808, 132

Ho I.-T., Kudritzki R.-P., Kewley L. J., Zahid H. J., Dopita M. A., Bresolin F., Rupke D. S. N., 2015, MNRAS, 448, 2030

Jones T., Ellis R. S., Richard J., Jullo E., 2013, ApJ, 765, 48

Kawata D., Gibson B. K., 2003, MNRAS, 340, 908

Kewley L. J., Ellison S. L., 2008, ApJ, 681, 1183

Kewley L. J., Geller M. J., Barton E. J., 2006, AJ, 131, 2004

Kewley L. J., Rupke D., Zahid H. J., Geller M. J., Barton E. J., 2010, ApJL, 721, L48

Kobayashi C., Springel V., White S. D. M., 2007, MNRAS, 376,1465

Krumholz M. R., 2013, MNRAS, 436, 2747

Lagos C. d. P., et al., 2015, MNRAS, 452, 3815

Lagos C. d. P., et al., 2018, MNRAS, 473, 4956

Lequeux J., Peimbert M., Rayo J. F., Serrano A., Torres-Peimbert S., 1979, A\&A, 80, 155

Ma X., Hopkins P. F., Wetzel A. R., Kirby E. N., AnglésAlcázar D., Faucher-Giguère C.-A., Kereš D., Quataert E., 2017, MNRAS, 467, 2430

Machado R. E. G., Tissera P. B., Lima Neto G. B., Sodré L., 2018, A\&A, 609, A66

Mackereth J. T., Crain R. A., Schiavon R. P., Schaye J., Theuns T., Schaller M., 2018, preprint, (arXiv:1801.03593)

Marigo P., 2001, A\&A, 370, 194

Marino R. A., et al., 2013, A\&A, 559, A114

Mast D., et al., 2014, A\&A, 561, A129

Matteucci F., Greggio L., 1986, A\&A, 154, 279

McAlpine S., et al., 2016, Astronomy and Computing, 15, 72

Michel-Dansac L., Lambas D. G., Alonso M. S., Tissera P., 2008, MNRAS, 386, L82

Molina J., Ibar E., Swinbank A. M., Sobral D., Best P. N., Smail I., Escala A., Cirasuolo M., 2017, MNRAS, 466, 892

Mollá M., Ferrini F., Díaz A. I., 1997, ApJ, 475, 519

Mollá M., Díaz Á. I., Gibson B. K., Cavichia O., López-Sánchez Á.-R., 2016, MNRAS, 462, 1329

Mollá M., Cavichia O., Costa R. D. D., Maciel W. J., Gibson B., Díaz A. I., 2017, in Liu X., Stanghellini L., Karakas A., eds, IAU Symposium Vol. 323, Planetary Nebulae: MultiWavelength Probes of Stellar and Galactic Evolution. pp 245253 (arXiv: 1612.03064), doi:10.1017/S1743921317001697

Mosconi M. B., Tissera P. B., Lambas D. G., Cora S. A., 2001, MNRAS, 325, 34

Obreja A., Domínguez-Tenreiro R., Brook C., Martínez-Serrano F. J., Doménech-Moral M., Serna A., Mollá M., Stinson G., 2013, ApJ, 763, 26

Oppenheimer B. D., Schaye J., Crain R. A., Werk J. K., Richings A. J., 2017, preprint, (arXiv:1709.07577)

Pedrosa S. E., Tissera P. B., 2015, A\&A, 584, A43

Perez M. J., Tissera P. B., Scannapieco C., Lambas D. G., de Rossi M. E., 2006, A $\$ \& \$ A, 459,361$

Perez J., Michel-Dansac L., Tissera P. B., 2011, MNRAS, 417, 580

Pilkington K., et al., 2012, MNRAS, 425, 969

Planck Collaboration et al., 2014, A\&A, 571, A1

Portinari L., Chiosi C., Bressan A., 1998, A\&A, 334, 505

Prantzos N., Boissier S., 2000, MNRAS, 313, 338

Queyrel J., et al., 2012, A\&A, 539, A93

Rosas-Guevara Y. M., et al., 2015, MNRAS, 454, 1038

Rupke D. S. N., Kewley L. J., Chien L.-H., 2010, ApJ, 723, 1255
Sales L. V., Navarro J. F., Theuns T., Schaye J., White S. D. M., Frenk C. S., Crain R. A., Dalla Vecchia C., 2012, MNRAS, 423,1544

Sánchez-Blázquez P., et al., 2014, A\&A, 570, A6

Sánchez-Menguiano L., et al., 2018, A\&A, 609, A119

Sánchez S. F., et al., 2013, A\&A, 554, A58

Sánchez S. F., et al., 2014, A\&A, 563, A49

Schaller M., Dalla Vecchia C., Schaye J., Bower R. G., Theuns T., Crain R. A., Furlong M., McCarthy I. G., 2015, MNRAS, 454,2277

Schaye J., 2004, ApJ, 609, 667

Schaye J., Dalla Vecchia C., 2008, MNRAS, 383, 1210

Schaye J., et al., 2010, MNRAS, 402, 1536

Schaye J., et al., 2015, MNRAS, 446, 521

Segers M. C., Schaye J., Bower R. G., Crain R. A., Schaller M., Theuns T., 2016, MNRAS, 461, L102

Sillero E., Tissera P. B., Lambas D. G., Michel-Dansac L., 2017, MNRAS

Springel V., 2005, MNRAS, 364, 1105

Stevens A. R. H., Lagos C. d. P., Contreras S., Croton D. J., Padilla N. D., Schaller M., Schaye J., Theuns T., 2017, MNRAS, 467, 2066

Swinbank A. M., Sobral D., Smail I., Geach J. E., Best P. N., McCarthy I. G., Crain R. A., Theuns T., 2012, MNRAS, 426, 935

Thielemann F., Nomoto K., Hashimoto M., 1993, in N. Prantzos, E. Vangioni-Flam, \& M. Casse ed., Origin and Evolution of the Elements. pp 297-309

Tissera P. B., 2000, ApJ, 534, 636

Tissera P. B., White S. D. M., Pedrosa S., Scannapieco C., 2010, MNRAS, 406, 922

Tissera P. B., White S. D. M., Scannapieco C., 2012, MNRAS, 420,255

Tissera P. B., Pedrosa S. E., Sillero E., Vilchez J. M., 2016, MNRAS, 456, 2982

Trayford J. W., et al., 2015, MNRAS, 452, 2879

Trayford J. W., et al., 2017, MNRAS, 470, 771

Troncoso P., et al., 2014, A\&A, 563, A58

White S. D. M., Rees M. J., 1978, MNRAS, 183, 341

Wiersma R. P. C., Schaye J., Smith B. D., 2009a, MNRAS, 393, 99

Wiersma R. P. C., Schaye J., Theuns T., Dalla Vecchia C., Tornatore L., 2009b, MNRAS, 399, 574

Yuan T.-T., Kewley L. J., Swinbank A. M., Richard J., Livermore R. C., 2011, ApJL, 732, L14

Zaritsky D., Kennicutt Jr. R. C., Huchra J. P., 1994, ApJ, 420, 87

Zavala J., et al., 2016, MNRAS, 460, 4466

\section{APPENDIX A: ASSESSMENT OF NUMERICAL RESOLUTION EFFECTS}

The analysis presented in this work is based on the galaxies identified in the $100 \mathrm{Mpc}$ cubic volume (Ref-L100N1504). The selected sample comprises gas discs with a large variety of $\nabla_{(\mathrm{O} / \mathrm{H})}$. As discussed in Section 2, a minimum number of particles has been adopted to diminish resolution problems in the estimation of the metallicity profiles. In order to further check the effects of resolution, we also estimate the $\nabla_{(\mathrm{O} / \mathrm{H})}$ in the high resolution run of $25 \mathrm{Mpc}$ box size, Recal-L025N0752 (see Table 1). This simulation has approximately an order of magnitude higher numerical resolution than Ref-L100N1504, allowing us to both check the robustness of the results against resolution and explore if smaller galaxies $\left(\mathrm{M}<10^{9.5} \mathrm{M}_{\odot}\right)$ follow the same trends.

We identify and measure the oxygen profiles of the disc components in Recal-L025N0752, applying the same criteria 
used for Ref-L100N1504, therefore extending the mass range down to $\sim 10^{9} \mathrm{M} \odot$. In Fig. A1, we show $\nabla_{(\mathrm{O} / \mathrm{H})}$ as a function of stellar mass (black line). The gradients have been rescaled by $-0.03 \mathrm{dex} \mathrm{kpc}^{-1}$. This is the difference estimated between the median simulated $\nabla_{(\mathrm{O} / \mathrm{H})}$ and the observed one by Belfiore et al. $(2017)$ at $\approx 10^{10.5} \mathrm{M}_{\odot}$. The simulated relation shows a trend consistent with observations in the sense that high mass galaxies tend to have gas discs with shallower $\nabla_{(\mathrm{O} / \mathrm{H})}$

To compare results from Recal-L025N0752 with those obtained for Ref-L100N1504, in Fig. A1 we also show the median relation for galaxies in Ref-L100N1504 in low density environment (cyan line; the relation has also been rescaled by -0.03 dex $\left.\mathrm{kpc}^{-1}\right)$. To improve the comparison, we estimate the local environment of galaxies in Recal-L025N0752, adopting the same stellar mass threshold used for RefL100N1504. We find that all selected galaxies in RecalL025N0752 belong to low density regions, $N_{C C} \leq 2$. Hence, the relations are consistent so that smaller galaxies show more negative metallicity gradients, although discs in RecalL025N0752 tend to have slightly flatter gradients.

\section{APPENDIX B: THE EFFECTS OF SN FEEDBACK}

The EAGLE project includes a set of simulations run with the same initial conditions in a $25 \mathrm{Mpc}$ box side volume, adopting different SN feedback energies: Ref-L025N0376, StrongFB-L025N0376 and WeakFB-L025N0376 (see Table 1 ). This set allows the assessment of the effects of varying the injected SN energy per event on the galaxy properties and, particularly, on the oxygen profiles. The variation of the energy injected by SNe has an impact on the gas fraction as well as on the stellar mass of galaxies as the star formation activity can be delayed to later times. Additionally, higher SN energy will also increase the impact of outflows triggered by starbursts. Both the regulation of the star formation activity and the transport of material out of the galaxy affect the chemical abundance patterns of the discs (Gibson et al. 2013).

The $\mathrm{D} / \mathrm{T}$ ratio can be also affected by variations in the SN feedback. This is relevant for our analysis since we are selecting discs with a minimum number of 1000 baryonic particles and 100 star-forming gas regions (Section 2). When these conditions are applied, only 9 galaxies are selected in WeakFB-L025N0376. This is probably the result of an earlier efficient transformation of gas into stars that did not leave enough material in the discs. In the case of StrongFBL025N037, as reported by Crain et al. (2017), galaxies have slightly larger HI gas mass at a given stellar mass. In Fig. $\mathrm{B} 1$, we show the $\mathrm{D} / \mathrm{T}$ distributions for the three runs. As can be seen, there are more galaxies dominated by dispersion in the WeakFB-L025N0376 and StrongFB-L025N0376 than in Ref-L025N0376.

It is also important to note that the galaxies in the RefL025N0376 have total and stellar disc sizes that correlate with stellar mass in agreement with observations (see also Furlong et al. 2015). However, the stellar discs in StrongFBL025N0376 have sizes with the opposite trends: high mass galaxies tend to have smaller stellar discs by a factor of approximately three compared to those in Ref-L025N0376 and WeakFB-L025N0376.

We estimate the depletion time (i.e. $\tau_{\text {Dep }}=\mathrm{M}_{\text {gas }} / \mathrm{SFR}$ ) as a function of stellar mass for the galaxies in the three runs. As can be seen from Fig. B1, there is trend for smaller galaxies to have longer depletion times regardless of SN energy adopted for feedback. However, at a given stellar mass, galaxies in StrongFB-L025N0376 are currently more efficiently forming stars. This is due to the high star-forming gas density (i.e. the combination of higher gas masses and small disc sizes).

For the purpose of our work, we are interested in assessing how the $\nabla_{(\mathrm{O} / \mathrm{H})}$ change with the SN feedback. In Fig. B2, we show the $\nabla_{(\mathrm{O} / \mathrm{H})}$ as a function of stellar mass for the three runs. WeakFB-L025N0376 have discs with $\nabla_{(\mathrm{O} / \mathrm{H})}$ that agree well with the observations as in the case of those in RefL025N0376. In the case of the StrongFB-L025N0376, there is a change in the relation: more positive $\nabla_{(\mathrm{O} / \mathrm{H})}$ are found in low mass galaxies (Fig. B2). The opposite trend can be seen in WeakFB-L025N0376. However, we acknowledge the fact that the number of galaxies in these samples are small.

The trends found in this analysis suggest that for enhanced SN feedback, there are more positive $\nabla_{(\mathrm{O} / \mathrm{H})}$ in smaller galaxies, in agreement with previous works (Gibson et al. 2013). This indicates that SN feecback is playing a role in shaping the relation between $\nabla_{(\mathrm{O} / \mathrm{H})}$ and stellar mass. Stronger feedback can delay the star formation to lower redshift (Crain et al. 2017), producing systems with more gas available to form stars at later times. On the other hand, smaller galaxies will experience stronger SN driven outflows because of the lower potential well, increasing the possibility to have positive gradients. 


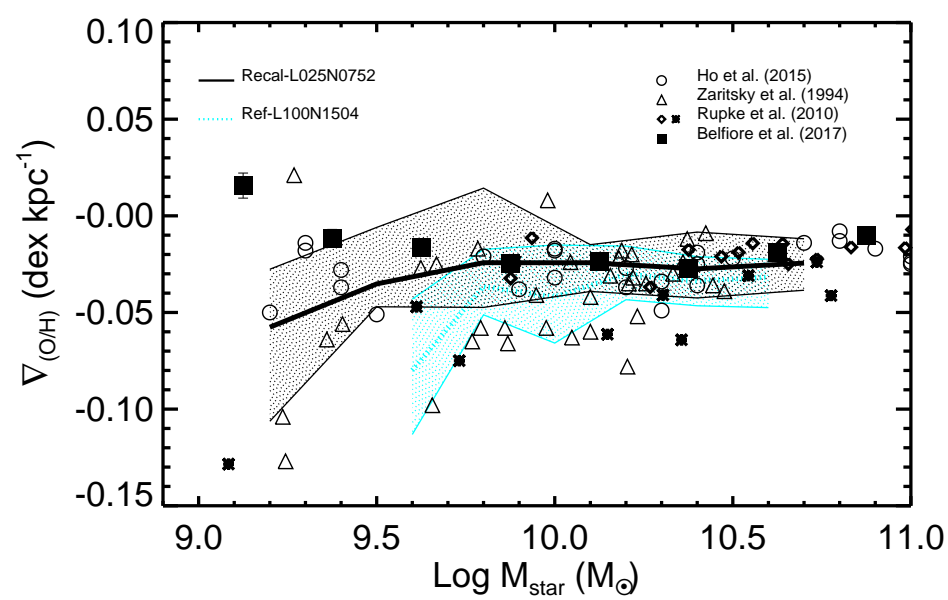

Figure A1. Median $\nabla_{(\mathrm{O} / \mathrm{H})}$ estimated for the star-forming disc components as a function of the stellar mass of the EAGLE galaxies in for the Recal-L025N0752 run (black solid lines). For comparison the simulated relation for the discs in low-density environments of the Ref-L100N1504 are also shown (cyan lines). The simulated relations have been rescaled by $-0.03 \mathrm{dex} \mathrm{kpc}^{-1}$ to match the mean observed values reported by Belfiore et al. $(2017)$ at $\approx 10^{10.5} \mathrm{M}_{\odot}$. The shaded area are defined by the first and the third quartiles.
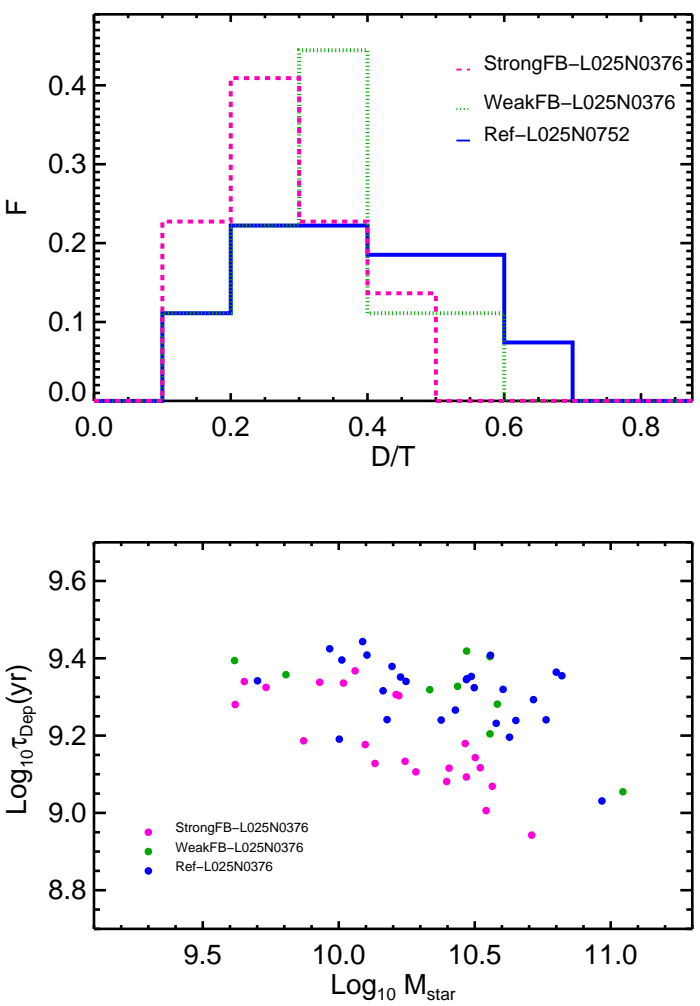

Figure B1. Upper panel: Mass-weighted distribution function of the $\mathrm{D} / \mathrm{T}$ ratios for the analysed galaxies in Ref-L025N0376 (blue solid line), WeakFB-L025N0376 (green dotted line) and StrongFB-L025N0376 (magenta dashed line). Lower panel: Depletion times as a function of stellar mass for the same set of galaxies.

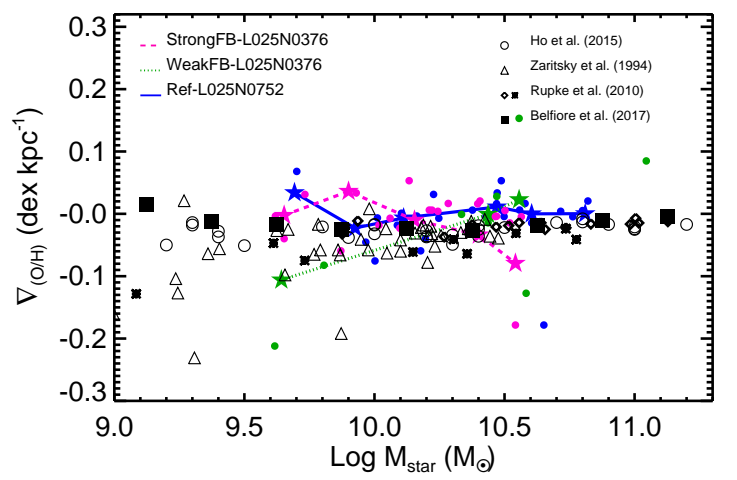

Figure B2. Median $\nabla_{(\mathrm{O} / \mathrm{H})}$ estimated for the star-forming disc components and the corresponding median values as a function of the stellar mass of the EAGLE galaxies in Ref-L025N0376 (blue solid line and circles), WeakFB-L025N0376 (green line and circles) and StrongFB-L025N0376 (magenta line and circles). For comparison observations have been included as in Fig. 3. 